\title{
Ohmic heating of asteroids around magnetic stars
}

\author{
Benjamin C. Bromley \\ Department of Physics 83 Astronomy, University of Utah, \\ 115 S 1400 E, Rm 201, Salt Lake City, UT 84112 \\ bromley@physics.utah.edu \\ Scott J. Kenyon \\ Smithsonian Astrophysical Observatory, \\ 60 Garden St., Cambridge, MA 02138 \\ skenyon@cfa.harvard.edu
}

\begin{abstract}
We consider the impact of electromagnetic induction and Ohmic heating on a conducting planetary object that orbits a magnetic star. Power dissipated as heat saps orbital energy. If this heat is trapped by an insulating crust or mantle, interior temperatures increase substantially. We provide a quantitative description of this behavior and discuss the astrophysical scenarios in which it might occur. Magnetic fields around some main-sequence stars and white dwarfs are strong enough to cause the decay of close-in orbits of asteroids and dwarf planets, drawing them through the Roche limit on Myr time scales. We confirm that Ohmic heating around neutron stars is driven by the rotation of the stellar magnetic dipole, not orbital dynamics. In any case, heating can raise interior temperatures of asteroids or dwarf planets on close-in orbits to well above liquidus. Hot material escaping to the surface may lead to volcanic ejections that can obscure the host star (as in the light curve of KIC 8462852) and pollute its atmosphere (as observed with metal-rich white dwarfs). We speculate that mixing of a volatile-rich mantle or crust with material from an induction-heated core may lead to an explosion that could destroy the asteroid prior to tidal break-up.
\end{abstract}

Subject headings: Planetary systems - Planets and satellites: formation - planet disk interactions 


\section{Introduction}

A magnetic star is a powerhouse. An asteroid passing close by experiences magnetic flux variations that drive an internal electric current. The interplay between this current, the magnetic field, and the asteroid's conductivity establishes how deep the magnetic flux penetrates into the asteroid. Resistive losses are inevitable. In the reference frame of the star, these losses come at the expense of the asteroid's kinetic energy. As the asteroid is heated, its orbit decays.

To explore this scenario, we rely on a quantitative description of the interaction between an idealized conducting sphere and a time varying magnetic field. Solutions to this problem have appeared in the literature for a wide range of applications. Bidinosti et al. (2007) provide a nice bibliographic overview, pointing out the early work of Mie, Debye (in scattering problems), Wait (geophysics), and Hoult and Lauterbur (NMR). More recently Ray et al. (2018) add in the effect of magnetic field gradients to generate a net force on the conductor as a method for sorting recyclable metals.

The ingredients for Ohmic heating are available in astrophysical settings. Iron or iron-nickel asteroids like (16) Psyche and planets with metallic cores like the Earth have regions of high conductivity. Hydrous or rocky planets may also be electrically conductive and able to sustain internal currents from electromagnetic induction. Examples of this phenomenon, sometimes called induction heating or Joule heating, include a conducting asteroid plowing through the magnetized plasma surrounding the young Sun or a T Tauri star (Sonett et al. 1970; Mckinnon 1989, Shimazu \& Terasawa 1995, Menzel \& Roberge 2013), unipolar induction through the moons of Jupiter and Saturn (Piddington \& Drake 1968; Goldreich \& Lynden-Bell 1969; Hand et al. 2011), and the heating of asteroids by the time-varying magnetic field of a pulsar (Cordes \& Shannon 2008; Kotera et al. 2016). Other applications include heating of conducting zones within hot Jupiters (Batygin et al. 2011; Wu \& Lithwick 2013, Ginzburg \& Sari 2016) and accretion flows (e.g. Bisnovatyi-Kogan

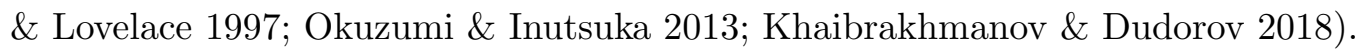

We focus here on a distinct mechanism for Ohmic heating driven by electromagnetic induction. When an asteroid orbits a magnetic star, it typically experiences a changing magnetic flux even if the stellar magnetic field is static in the star's rest frame. We expect strong flux variations for highly eccentric orbits when the periastron is near the Roche limit, as when a comet or asteroid is scattered toward the stellar host by a more distant planet (e.g., Rasio \& Ford 1996; Chatterjee et al. 2008). The resulting electromotive force drives electrical currents within a conducting asteroid, generating Ohmic heat. The net magnetic force on the eddy currents produces a drag, causing a loss of speed and orbital energy. The amount of energy drawn per orbit, as we quantify below, is small compared to the energy in the star's magnetic field. Thus, any back-reaction of the asteroid's slow orbital evolution onto the star and its magnetic dynamo is inconsequential; all of the energy dissipated by induced currents comes from the loss of orbital energy. 
Ohmic dissipation in a conducting asteroid contributes to its overall thermal evolution. Ohmic heating takes place in specific regions of the asteroid, for example only in a conducting core, and then only near the core's surface if the skin effect limits the magnetic penetration into the conducting medium. Ohmic heat thus serves as a localized energy source, contributing to the heat flow throughout the asteroid. Other factors include stellar illumination and radiative cooling at the surface, along with the asteroid's physical and material characteristics. Thermal time scales are rapid compared to orbital evolution; small asteroids may reach a steady state quickly, with energy input from starlight and Ohmic heating balanced by radiative cooling. A larger asteroid with a conducting core that is thermally insulated by a deep mantle may undergo runaway core heating. The diffusion equation quantifies this process, helping to distinguish among hypothetical possibilities.

These outcomes require close-in orbits around stars with strong magnetic fields. Magnetic pre-main-sequence stars and some late-type dwarfs have fields as strong as $B_{*} \sim 10^{3} \mathrm{G}$ on the stellar surface (e.g., Johns-Krull et al. 2009). Some Ap stars, such as HD 215441 (Babcock 1960) and HD 75049 (Elkin et al. 2010), have much stronger fields $\left(B_{*} \gtrsim 30 \mathrm{kG}\right)$. A few magnetic white dwarfs have surface field strengths approaching $10^{9}$ G (e.g. Angel et al. 1981; Schmidt \& Smith 1995 Wickramasinghe \& Ferrario 2000; Ferrario et al. 2015). Neutron stars can achieve field strengths as high as $10^{15} \mathrm{G}$ (Kaspi \& Beloborodov 2017 provide a review). Close-in orbits around these stars are also plausible, as they have been observed directly in exoplanet searches (Sanchis-Ojeda et al. 2014a; KOI 1843.03 has the shortest known orbital period, $4.245 \mathrm{hr}$ around a red dwarf; Ofir \& Dreizler 2013: Rappaport et al. 2013). Close-in planetary bodies are also inferred from the presence of metals in white dwarf atmospheres (e.g., Jura 2006, Farihi et al. 2010) as well as transiting dust clouds (e.g., WD 1145+017; Alonso et al. 2016).

Here we consider if electromagnetic induction within orbiting bodies can sap orbital energy at a rate large enough and generate enough heat to have observable consequences in real astrophysical systems. First, we describe electromagnetic induction in a spherical conductor $(\$ 2)$, and the impact of induced currents on its orbit $(\$ 3)$ and its thermal evolution $(\$ 4)$. Then we discuss how this phenomenon might play out in several astrophysical scenarios (\$5). We summarize our results (\$6).

\section{Electromagnetic induction}

An electrically conducting asteroid, or one with a conducting core, experiences an oscillating magnetic field as it orbits a magnetic star. To calculate the Ohmic heating from eddy currents that draw energy from orbital motion, we first consider the case of an idealized conductor sitting at rest in a time varying magnetic field. This situation gives the response of the asteroid to one Fourier

mode of the time-varying field, as seen in the asteroid's rest frame. We then apply the results to the situation where time variations correspond to full orbital motion through the stellar field. 


\subsection{Ohmic heating in a time-varying uniform magnetic field}

In an idealized model, an asteroid is a sphere of radius $r_{\text {ast }}$ and mass $m$, with constant, realvalued electrical conductivity $\sigma$ and magnetic permeability $\mu$. We assume that $r_{\text {ast }}$ is small, so that the stellar magnetic field $\vec{B}$ in its vicinity is, to a good approximation, spatially uniform. Then, $\vec{B}=B_{0} \exp (-i \omega t) \hat{e}_{z}$, where the field is aligned with the $z$-axis in the asteroid's reference frame, and $\omega$ is an angular frequency characteristic of orbital motion. This frequency is low enough that we may safely ignore electromagnetic scattering ( $\omega \epsilon \ll \sigma$, where $\epsilon$ is the permittivity; see Kotera et al. 2016). The induced electric field $\vec{E}$ and current density $\vec{J}$ are related through Ohm's Law $(\vec{J}=\sigma \vec{E})$; thus, we can use Maxwell's equations to solve for $\vec{B}$ and $\vec{J}$ directly, deriving the Ohmic heating from $|\vec{J}|^{2} / \sigma$.

Motivated by research in magnetic resonance imaging, Bidinosti et al. (2007) obtain an elegant solution for Ohmic power loss in the problem of a homogeneous conducting magnetic sphere in a time varying magnetic field, extending earlier work that considered non-magnetic media. In their application, the field variations are rapid (radio frequencies) and the spheres are small (centimeters), but their solution is general, applying equally well to orbital time scales and kilometer-size objects 1 By matching boundary conditions for the magnetic field at large distances and at the sphere's surface, they show that the current density within the asteroid lies in the azimuthal direction with magnitude

$$
J(r, \theta)=\frac{3 \pi B_{0}|k|^{2} r\left[j_{0}(k r)+j_{2}(k r)\right] \sin (\theta)}{2\left[\left(\mu+2 \mu_{0}\right) j_{0}\left(k r_{\text {ast }}\right)+\left(\mu-\mu_{0}\right) j_{2}\left(k r_{\text {ast }}\right)\right]},
$$

where $r$ and $\theta$ are spherical polar coordinates with an origin at the asteroid's center, $k=\sqrt{i \mu \sigma \omega}$, $\mu_{0}$ is the vacuum permeability, and

$$
\begin{aligned}
& j_{0}(z)=\sin (z) / z \\
& j_{2}(z)=\left(3 / z^{3}-1 / z\right) \sin (z)-3 \cos (z) / z^{2}
\end{aligned}
$$

are spherical Bessel functions $2^{2}$ In this Fourier analysis, the (complex) current density oscillates as $\exp (-i \omega t)$.

A quantity that characterizes the current density is the skin depth $\delta=\sqrt{2 / \mu \sigma \omega}$, which gives an indication where in the sphere the current is strong. In the limit of low frequency, for example, $\delta$ can be large, exceeding the conductor's size. Then the current is spread over much of the asteroid's interior. At high frequencies, $\delta$ is small, and current is confined to the surface of the sphere.

\footnotetext{
${ }^{1}$ We follow Bidinosti et al. (2007) in adopting the SI system for Maxwell's Equations and derived quantities. We use mixed units when giving characteristic values of these and related quantities, according to typical uses in the literature.

${ }^{2}$ When evaluating the spherical Bessel functions with complex arguments, we use the forms with sines and cosines, for which modern programming languages (python and $\mathrm{C}++$ ) support complex arithmetic.
} 
The average power lost to Ohmic heating in the asteroid is

$$
P_{\mathrm{Ohm}}=\frac{1}{2} \int_{\text {sphere }} \frac{|J(r, \theta)|^{2}}{\sigma} d V=\frac{2 \pi r_{\mathrm{ast}}^{2} B_{0}}{\mu_{0}} \operatorname{Im}\left\{\frac{J\left(r_{\mathrm{ast}}, \pi / 2\right)}{i \sigma}\right\}
$$

where the rightmost expression, from Bidinosti et al. (2007, Eq. 20 therein), is the product of the magnetic flux through the sphere's midplane and the electric field strength at its equator. Bidinosti et al. (2007) identified it for the case of non-magnetic media $\left(\mu=\mu_{0}\right)$, and we confirm that the expression holds for magnetic material $\left(\mu>\mu_{0}\right)$. With this result we can write the power loss as the product of the average magnetic energy density, the rate at which the magnetic field changes, the volume of the sphere, and an "efficiency factor" $\mathcal{F}$ that depends only on dimensionless quantities:

$$
\begin{aligned}
P_{\mathrm{Ohm}} & =\frac{B_{0}^{2}}{2 \mu_{0}} \omega \frac{4 \pi r_{\text {ast }}^{3}}{3} \mathcal{F}\left(R_{m}, \mu_{\text {rel }}\right) \\
\mathcal{F}\left(R_{m}, \mu_{\text {rel }}\right) & \equiv \operatorname{Im}\left\{\frac{9 \mu_{\text {rel }}\left[j_{0}(z)+j_{2}(z)\right]}{2\left[\left(\mu_{\text {rel }}+2\right) j_{0}(z)+\left(\mu_{\text {rel }}-1\right) j_{2}(z)\right]}\right\},
\end{aligned}
$$

where $\mu_{\text {rel }} \equiv \mu / \mu_{0}$ and $R_{m}=\mu \sigma \omega r_{\text {ast }}^{2}$ are the relative permeability and magnetic Reynolds number, respectively, and $z \equiv \sqrt{i R_{m}}$. The Reynolds number is related to the skin depth through $\delta=$ $r_{\text {ast }} \sqrt{2 / R_{m}}$. In general, $0 \leq \mathcal{F}<1$.

The efficiency factor $\mathcal{F}\left(R_{m}, \mu_{\text {rel }}\right)$ encodes the detailed physics of electromagnetic induction and Ohmic heating. Its behavior falls into three distinct regimes, depending on the values of the Reynolds number and the relative permeability:

I. When the Reynolds number is low, $R_{m} \lesssim 3$, the efficiency factor scales as $\mathcal{F} \sim R_{m}^{2}$. The local stellar magnetic field permeates through the conducting asteroid, dominating over the induced field. The skin depth is comparable to the radius of the sphere or larger, indicating that an eddy current forms within the bulk material.

II. At intermediate Reynolds number, $3 \lesssim R_{m} \lesssim \mu_{\text {rel }}^{2}$, the efficiency factor scales as $\mathcal{F} \sim R_{m}^{1 / 2}$. The current density is concentrated near the asteroid's surface but magnetization from the bulk material also responds to the applied field. This regime applies only to magnetic material.

III. At high Reynolds number, $R_{m} \gtrsim \mu_{\text {rel }}^{2}$, the efficiency factor falls off as $\mathcal{F} \sim R_{m}^{-1 / 2}$. The current density is nearly an ideal surface current, $K=J \delta$, which cancels the varying magnetic field in the interior of the asteroid independently of any bulk magnetization.

If the conducting medium is non-magnetic $\left(\mu_{\text {rel }} \sim 1\right)$, then the efficiency transitions from regime I directly to regime III. The efficiency factor reaches a peak of approximately $53 \%$ at the transition point, near $R_{m}=11.6$. In high-permeability material $\left(\mu_{\text {rel }} \gg 1\right)$, the efficiency factor has a maximum that is just above $93 \%$ at $R_{m}=\mu_{\text {rel }}^{2}$. Figure 1 provides an illustration, showing efficiency curves $\left(\mathcal{F}\right.$ versus $\left.R_{m}\right)$ for several values of $\mu_{\text {rel }}$. 


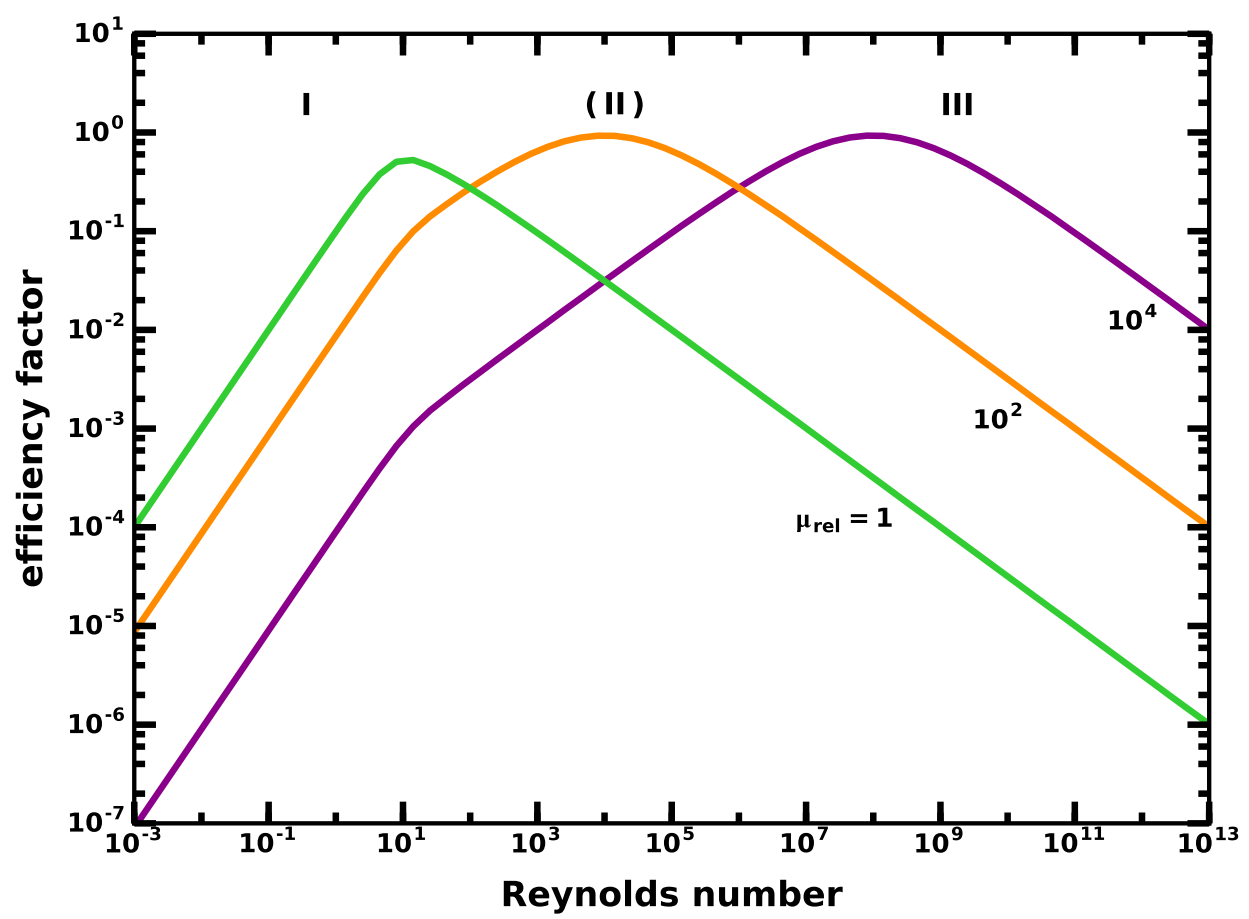

Fig. 1. - The efficiency factor as a function of magnetic Reynolds number for a spherical conductor. At low $R_{m}$, labeled as regime I, the applied (stellar) magnetic field dominates throughout the medium and the current density builds up in the bulk of the conductor. The efficiency factor varies as $R_{m}^{2}$. In region II, applicable only to magnetic media with relative permeability $\mu_{\text {rel }}>1$, the efficiency factor scales as $R_{m}^{1 / 2}$ as a result of the contributions of the magnetization in response to the changing magnetic flux. Regime III corresponds to $R_{m}>\mu_{\text {rel }}^{2}$. There the efficiency factor declines as $R_{m}^{-1 / 2}$. In this regime, the surface current eliminates the applied field inside the conductor.

Expressions of the power loss in the three regimes are

$$
\begin{aligned}
P_{I} & \approx \frac{3 \pi}{5} B^{2} \mu_{\text {rel }}^{2}\left(\mu_{\mathrm{rel}}+2\right)^{-2} \sigma \omega^{2} r_{\text {ast }}^{5} & & \left(R_{m} \lesssim 3\right) \\
P_{I I} & \approx \frac{2}{3} B^{2}\left(\mu_{0} \mu_{\mathrm{rel}}\right)^{-1 / 2} \sigma^{1 / 2} \omega^{3 / 2} r_{\mathrm{ast}}^{4} & & \left(3 \lesssim R_{m} \lesssim \mu_{\mathrm{rel}}^{2}\right) \\
P_{I I I} & \approx 3 \pi B^{2}\left(\mu_{\mathrm{rel}} / \mu_{0}^{3}\right)^{1 / 2} \sigma^{-1 / 2} \omega^{1 / 2} r_{\mathrm{ast}}^{2} & & \left(R_{m} \gtrsim \mu_{\mathrm{rel}}^{2}\right) .
\end{aligned}
$$

With ferromagnetic material, a complicating factor is that the relative permeability drops to unity when the temperature increases beyond the Curie temperature $T_{\text {Curie }}$, around $10^{3} \mathrm{~K}$ for pure iron. We discuss this effect below. We do not consider other phenomena associated with ferromagnets, such as hysteresis.

If we fix the bulk material properties of an asteroid, along with the frequency $\omega$, we can identify 
an optimal radius that maximizes the efficiency $\mathcal{F}$ :

$$
r_{\mathrm{opt}} \sim \begin{cases}3.4 / \sqrt{\mu_{0} \sigma \omega} & \left(\mu_{\mathrm{rel}} \sim 1\right) \\ \sqrt{\mu_{\mathrm{rel}} / \mu_{0} \sigma \omega} & \left(\mu_{\mathrm{rel}} \gg 1\right),\end{cases}
$$

The upper equation is appropriate to solids made of ice, water or rock, while the lower equation is for solid ferromagnetic asteroids or their cores. In all cases, the frequency $\omega$ corresponds to an oscillatory mode of the magnetic field. Multiple modes may contribute to the heating process; which ones are determined by the time variation in the magnetic field as the asteroid orbits its stellar host.

As an example, a metallic solid orbiting in the dipole field of the Sun at $0.1 \mathrm{AU}$, the optimal radius for Ohmic heating is roughly a kilometer. For a rocky asteroid, $r_{\text {opt }}$ can exceed a $100 \mathrm{~km}$. The precise value of $r_{\text {opt }}$ depends on material properties, the nature of the stellar magnetic field, and how an orbiting body moves through the field. These topics are next.

\subsection{Orbital motion in the dipole magnetic field}

When the stellar field is a dipole, the magnetic field at a position $\vec{d}$ relative to the star is

$$
\vec{B}=B_{*} \frac{R_{*}^{3}}{d^{3}}\left[3\left(\hat{e}_{r} \cdot \hat{e}_{z}\right) \hat{e}_{r}-\hat{e}_{z}\right],
$$

where $B_{*}$ specifies the field strength at the surface of the star at radius $R_{*}$, and the unit vector $\hat{e}_{z}$ is aligned with the star's magnetic dipole moment $\vec{m}_{*}$. We assume that the magnetic field in the star's rest frame is static, with fixed orientation. We further designate that a reference orbital plane lies perpendicular to $\vec{m}_{*}$; orbits in this plane have inclination $i=0^{\circ}$. Additionally we assume that the effect of the magnetic field on an orbiting solid is small compared to gravity, so that its osculating orbit is Keplerian with mean angular velocity $\Omega_{a}=\sqrt{G M_{*} / a^{3}}$, where $M_{*}$ is the stellar mass and $a$ is the semimajor axis.

The idealization of a static dipole field carries the assumption that the asteroid's orbital motion is fast compared with the rotation of the star or that the dipole moment is closely aligned with the star's angular momentum (or both). While this approximation is not valid for pulsars, it is reasonable for main-sequence stars and most white dwarfs.

In the asteroid's reference frame, a static magnetic field is perceived as time varying, provided that the asteroid has either some eccentricity $e>0$ or inclination $i>0^{\circ}$. Two limiting cases are (i) a circular "polar" orbit of radius $a$ in a plane containing the stellar magnetic dipole moment $\left(i=90^{\circ}\right)$ and (ii) a highly eccentric "equatorial" orbit in the fiducial orbital plane $\left(i=0^{\circ}\right)$. In the first case, the magnetic field strength variations are predominantly sinusoidal, with an angular frequency that is twice the Keplerian value $\Omega_{a}$, since north and south poles are equivalent in terms 
of the strength of the changing magnetic field. In this preliminary work, we focus only on the field strength, ignoring changes in the alignment of the magnetic field. The approximate orbit-averaged power loss is then given by Equation (5) with $\omega=2 \Omega_{a}$.

In the case of an eccentric orbit with semimajor axis $a$ and periastron distance $q$, we estimate the Ohmic power loss by first approximating the inner part of an asteroid's orbit using a parabolic trajectory with the same periastron distance. We then decompose the magnetic field sampled along this route into its frequency components with an FFT of $B(d(t))$ over a time interval $T$, set to be about 10 times the circular orbital period at the periastron distance. We find that the spectrum of $B(\omega)$ is well-approximated as an exponential with decay constant $v_{q} / q$, where $v_{q}$ is the asteroid's speed at periastron. Accordingly, we estimate power loss during periastron passage using Equation (5) at characteristic frequency $\omega=\sqrt{2} \Omega_{q} \approx v_{q} / q$, where $\Omega_{q}=\sqrt{G M_{*} / q^{3}}$ is the angular frequency of a circular orbit at distance $q$. Multiplying this power loss by the duration of passage, $2 q / v_{q}$, yields the energy loss per orbit. Comparison with calculations using the full spectrum suggests that this approximation is useful when the eccentricity of the orbit is $e \gtrsim 0.4$.

To summarize the results just described, the orbit-averaged Ohmic power loss experienced by an asteroid is

$$
\left\langle P_{\mathrm{Ohm}}\right\rangle \approx \frac{2 r_{\mathrm{ast}}^{3}}{3} \frac{B_{*}^{2}}{\mu_{0}} \Omega_{a} \mathcal{F}\left(R_{m}, \mu_{\mathrm{rel}} ; \omega\right) \times \begin{cases}2 \pi R_{*}^{6} / a^{6} & \left(\text { circular polar; } \omega=2 \Omega_{a}\right) \\ R_{*}^{6} / q^{6} & \left(\text { eccentric, } 0.4 \lesssim e<1 ; \omega=\sqrt{2} \Omega_{q}\right),\end{cases}
$$

where the Reynolds number is evaluated with the angular frequency $\omega=2 \Omega_{a}$ and $\sqrt{2} \Omega_{q}$ for circular polar orbits $\left(i=90^{\circ}\right)$ and eccentric equatorial orbits $\left(i=0^{\circ}\right)$, respectively. In general, objects with a size near $r_{\text {opt }}$ (Eq. (10) ) experience the greatest amount of heating per unit mass. We focus first on these objects when considering whether "orbit-induced" Ohmic heating might play a role in astrophysical scenarios.

\section{Orbital evolution}

An asteroid moving through the magnetic field of its stellar host feels a Lorentz force as a result of interactions between the induced currents within it and the stellar field. While the detailed dynamics can be complicated, depending on the nature of the field gradients and the orbit geometry, our interest is on how the overall orbit changes with time. Our focus is specifically on orbital energy, as it is most connected to Ohmic heating.

For the effects described here to be important, an asteroid must transit rapidly through a region of strong magnetic field, close to its host. If the asteroid is to survive the stellar tidal forces 
during the encounter, it must not venture too far inside the Roche limit,

$$
R_{\text {Roche }}=k\left(\frac{M_{*}}{\bar{\rho}}\right)^{1 / 3} \approx 0.86 k\left[\frac{M_{*}}{0.5 \mathrm{M}_{\odot}}\right]^{1 / 3}\left[\frac{\bar{\rho}}{5 \mathrm{~g} / \mathrm{cm}^{3}}\right]^{-1 / 3} \mathrm{R}_{\odot},
$$

where $\bar{\rho}$ is the average mass density of the conducting asteroid and the parameter $k$ is a constant of order unity. A value of $k=0.8$ corresponds to the case where the asteroid is a strengthless, non-rotating solid body. Rotating objects have larger $k$, as do fluid bodies; objects with significant tensile strength have smaller $k$ (e.g., Veras et al. 2017, see also Davidsson 1999; Jura|2003; Holsapple \& Michel 2008).

For the value of $k=1$ adopted here, the Roche radius lies outside of the stellar surface for stars that are more dense than the Sun, including red dwarfs, white dwarfs and neutron stars. For less dense stars with $R_{\text {Roche }}<R_{*}$, which include main-sequence stars that are more massive than the Sun, we consider star-grazing orbits instead.

With the Roche limit (Eq. (13)) as a guide, we estimate the optimal physical radius of an asteroid for efficient Ohmic heating (Eq. 10). Around a late-type star or white dwarf,

$$
\begin{array}{rlrl}
r_{\mathrm{opt}} & \sim\left(\frac{\mu_{\mathrm{rel}}}{\mu_{0} \sigma}\right)^{1 / 2}\left(\frac{q^{3}}{G M_{*}}\right)^{1 / 4} \\
& \approx 3\left[\frac{\mu_{\mathrm{rel}}}{5000}\right]^{1 / 2}\left[\frac{\sigma}{10^{6} \mathrm{~S} / \mathrm{m}}\right]^{-1 / 2}\left[\frac{M_{*}}{0.5 \mathrm{M}_{\odot}}\right]^{-1 / 4}\left[\frac{q}{1 \mathrm{R}_{\odot}}\right]^{3 / 4} \mathrm{~km} & & \text { (iron/nickel) }, \\
& \approx 64\left[\frac{\sigma}{5 \mathrm{~S} / \mathrm{m}}\right]^{-1 / 2}\left[\frac{M_{*}}{0.5 \mathrm{M}_{\odot}}\right]^{-1 / 4}\left[\frac{q}{1 \mathrm{R}_{\odot}}\right]^{3 / 4} \mathrm{~km} & \text { (water) }, \\
& \approx 1400\left[\frac{\sigma}{0.01 \mathrm{~S} / \mathrm{m}}\right]^{-1 / 2}\left[\frac{M_{*}}{0.5 \mathrm{M}_{\odot}}\right]^{-1 / 4}\left[\frac{q}{1 \mathrm{R}_{\odot}}\right]^{3 / 4} \mathrm{~km} & \text { (rock) } .
\end{array}
$$

The numerical values of $\mu_{\text {rel }}$ and $\sigma$ are order-of-magnitude estimates for solid iron in a ferromagnetic asteroid (e.g., de Koker et al. 2012) and liquid water in a "water world" (Thomas et al. 1934). The conductivity of rocky, planetary material can vary enormously, from $\sim 10^{-5} \mathrm{~S} / \mathrm{m}$ to $O(1) \mathrm{S} / \mathrm{m}$ (e.g., Schwarz 1990). Here, we adopt an intermediate value of $0.01 \mathrm{~S} / \mathrm{m}$ (cf. Xu et al. 1998).

The numerical reference values in Equations (15)-(17) also reflect the angular frequency of magnetic flux variation, which arises from orbital motion. The reference values are based on a close-in, circular orbit about a red dwarf host. If the orbit were eccentric, but with the same periastron distance, the optimal radius would increase. Still the main frequency component of the magnetic flux variations would be similar, reflecting the fact that $\omega \sim v / q$ where the field is strongest. Thus, for a given periastron distance $q$ we use the same $r_{\text {opt }}$ value when considering circular and eccentric orbits.

Generally, reducing the orbital distance reduces $r_{\mathrm{opt}}$. The optimal radius also shrinks as the stellar mass increases, so long as the asteroid's orbit is close to star-grazing. 
Next, we consider specific orbital configurations for which Ohmic heating can play a role. Circular polar orbits perpendicular to the magnetic dipole moment lead to inspiral, while eccentric orbits that cut in and out of the field in the plane perpendicular to $\vec{m}_{*}$ experience eccentricity damping at approximately fixed periastron distance. In most astrophysical situations, the relative orientation of asteroid orbits falls somewhere between these two idealizations.

\subsection{Circular inspiral}

As Ohmic heating draws orbital energy, the orbit of an asteroid at semimajor axis $a$ decays at a rate of

$$
\frac{d a}{d t}=-\frac{2 a^{2}\left\langle P_{\mathrm{Ohm}}\right\rangle}{G M_{*} m}
$$

where the orbit-averaged Ohmic heating rate $\left\langle P_{\mathrm{Ohm}}\right\rangle$ is given in Equation (12). To explore the possibilities of orbital decay from Ohmic heating, we focus on asteroids with radii comparable to $r_{\text {opt }}$ (Eq. (14)), and on a circular polar orbit near the Roche limit. If the efficiency factor does not change substantially as the asteroid spirals inward, $d a / d t \sim-a^{-11 / 2}$. The formal solution to this ODE yields a time scale for the inspiral,

$$
\begin{aligned}
\tau_{\text {inspiral }} & \approx \frac{1}{13} \bar{\rho}\left(G M_{*}\right)^{1 / 2} \frac{\mu_{0} a^{13 / 2}}{R_{*}^{6} B_{*}^{2}} \mathcal{F}^{-1} \\
& \approx 0.84\left[\frac{\bar{\rho}}{5 \mathrm{~g} / \mathrm{cm}^{3}}\right]\left[\frac{M_{*}}{0.5 \mathrm{M}_{\odot}}\right]^{1 / 2}\left[\frac{R_{*}}{0.5 \mathrm{R}_{\odot}}\right]^{-6}\left[\frac{B_{*}}{5 \mathrm{kG}}\right]^{-2}\left[\frac{a}{1 \mathrm{R}_{\odot}}\right]^{13 / 2} \mathcal{F}^{-1} \mathrm{Myr}
\end{aligned}
$$

where the power-law indices show how parameters scale for an asteroid with radius near $r_{\text {opt }}$ and the numerical reference values are plausible for a magnetic red dwarf. This time scale suggests that orbital decay by Ohmic heating is possible in an astrophysical setting.

This analysis carries the assumption that an asteroid remains close to optimal size as its orbit evolves. In actuality, the efficiency factor for Ohmic heating changes as the body moves inward. Nonetheless, the asteroid's inspiral rate will continue to get faster as inspiral proceeds. For example, when $\mathcal{F}$ shifts away from its peak into regime III (e.g., Fig. 1), the inspiral rate still increases, with the orbital distance decreasing as $d a / d t \sim-a^{-4}$.

The time scale for orbital decay in Equation (19), with numerical values tuned for a red dwarf, is similar for white dwarfs and early-type magnetic stars. In all cases, the asteroid needs to be within a Solar radius or so of its stellar host to experience "magnetic migration". Bodies larger than roughly a kilometer, which are bound by gravity, have a Roche limit that is comparable to this distance. They cannot inspiral much before being tidally disrupted. Small bodies with high tensile strength $\left(r_{\text {ast }} \ll 1 \mathrm{~km}\right)$ can orbit closer to the host star than larger ones (e.g., Kenyon \& Bromley 2017a). However, so close to the star there may be competition between Ohmic heating and other effects like radiative heating. 


\subsection{Eccentricity damping}

An asteroid on an eccentric orbit also loses orbital energy to Ohmic heating. Most of this dissipation takes place near periastron, where kinetic energy is lost with no significant deflection from a Keplerian path. Thus, with each close passage, the asteroid slows incrementally, keeping its periastron distance fixed while shaving off apoastron distance at the expense of its semimajor axis. In this way the asteroid's eccentricity damps as its semimajor axis falls. In the final stages of circularization of an asteroid in the equatorial plane, the decay of the semimajor axis slows because the magnetic field variations encountered by the asteroid become less significant, despite that the field itself is comparatively strong there. Then the asteroid damps onto a circular orbit just beyond the initial periastron. If the asteroid has a non-zero inclination, it will always encounter magnetic field variations along its orbit. It thus settles onto a circular orbit and inspirals toward the host star.

To demonstrate this effect, we use the $n$-body routine in our Orchestra code (e.g., Bromley \& Kenyon 2011), modified to include the loss of kinetic energy from Ohmic heating. We choose an asteroid that has a radius below optimal size, $r_{\text {ast }}<r_{\text {opt }}\left(R_{m} \lesssim 1\right.$; regime I), so that the magnetic field is locally unperturbed by the asteroid's eddy currents. The instantaneous force on the asteroid then can be estimated directly from Maxwell's equations (van Bladel 1988; Giffin et al. 2010), yielding

$$
\vec{F}=-\frac{2 \pi \sigma r_{\text {ast }}^{5}}{15} \vec{v}\left|\left(\hat{e}_{v} \cdot \vec{\nabla}\right) \vec{B}\right|^{2} \quad\left(R_{m} \lesssim 1\right)
$$

where $\vec{v}$ is the asteroid's velocity, $\hat{e}_{v} \equiv \vec{v} / v$, and $\vec{B}$ is the local value of the stellar magnetic field. This force acts as a drag, opposing the asteroid's velocity. The strong dependence on radial distance $\left(d^{-17 / 2}\right)$, as well as orientation (the gradient in the magnetic field strength vanishes at periastron when $i=0^{\circ}$ ), indicates that the force on the asteroid varies widely along its orbit. If the orbital eccentricity is modestly high, the asteroid gets the strongest kicks just before and after it approaches periastron.

Figure 2 illustrates orbital evolution with the force law in Equation 21) using Orchestra. The figure shows periastron, semimajor axis, and apoastron of a $250 \mathrm{~km}$ rocky asteroid on a close-in eccentric orbit around a red dwarf host. Here, we artificially increase the stellar magnetic field by four orders of magnitude above observed values for red dwarfs so that we limit the number of orbits we need to integrate to measure the orbital evolution. In the figure, we see that eccentricity damping is a general feature, independent of the asteroid's orbital inclination. However, if the asteroid is in the equatorial plane, its orbit stops evolving after it circularizes - the magnetic field is constant in its frame, and there is no Ohmic heating. If the orbit is inclined, then the asteroid always experiences some field variations; circular inspiral is inevitable, as in 93.1 .

Here we focus on the orbital evolution of a highly eccentric asteroid, before it circularizes. The average Ohmic power loss of an asteroid on an eccentric orbit at fixed periastron distance scales 


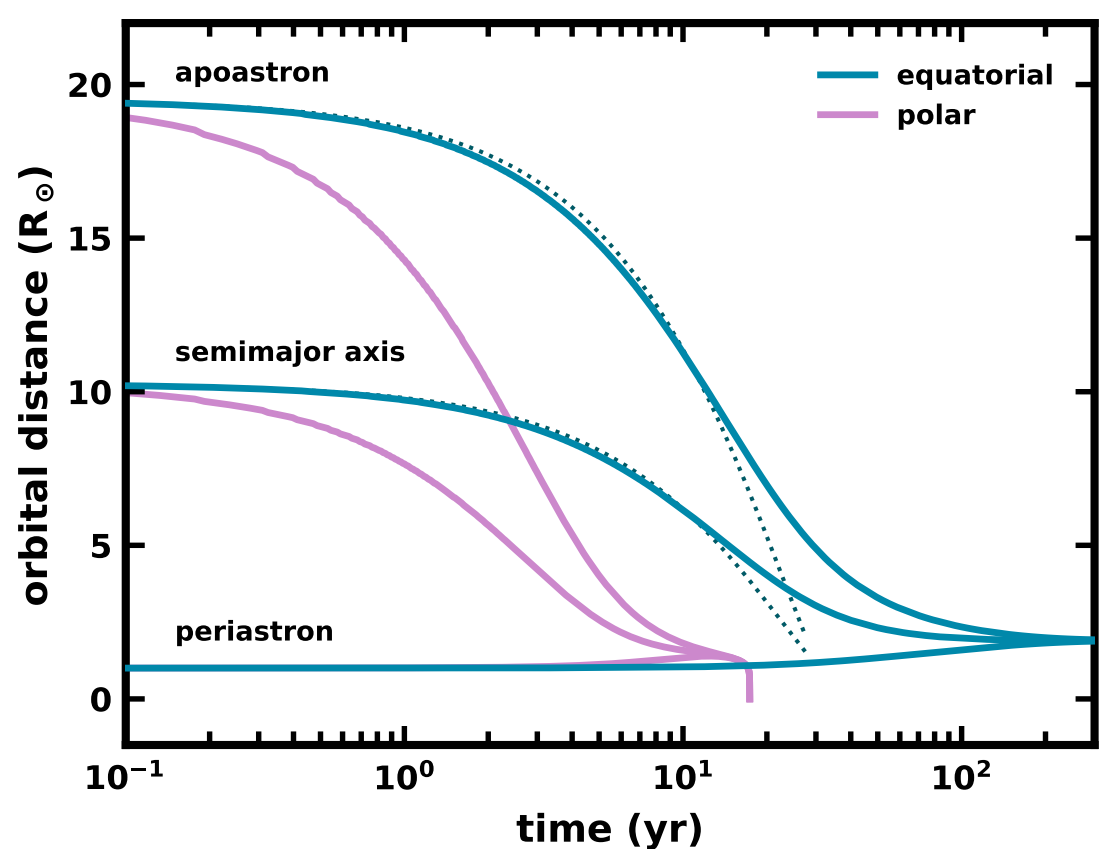

Fig. 2.- Simulation of a large non-magnetic rocky asteroid $\left(r_{\text {ast }}=250 \mathrm{~km}, \rho=3 \mathrm{~g} / \mathrm{cm}^{3}\right.$, $\sigma=0.01 \mathrm{~S} / \mathrm{m})$, around a red dwarf $\left(R_{*}=0.5 \mathrm{R}_{\odot}, M_{*}=0.5 \mathrm{M}_{\odot}\right)$ with an unrealistically high magnetic field $\left(B_{*}=5 \times 10^{7} \mathrm{G}\right)$ to reduce the computational run time needed to observe orbital evolution. The blue-green curves are for an orbit in the equatorial plane (perpendicular to the stellar magnetic dipole), the solid curve is from a simulation while the dotted curve is the theoretical expectation. The eccentricity damps at nearly fixed periastron until the asteroid's orbit gets close to circular. Because the magnetic field is constant at fixed orbital distance, this asteroid will settle on circular orbit. The magenta curves are for an asteroid on a polar orbit; the stronger field variations experienced by the asteroid on that orbit yield a faster damping time. Furthermore, those field variations remain even after circularization, hence the rapid plummet in the orbital distance once the eccentricity reaches zero. 
with semimajor axis as $a^{-3 / 2}$. This result stems from Equation 12, which accounts for the brief, periodic loss of orbital energy near periastron at a rate governed by the orbital frequency. Ohmic dissipation translates to eccentricity damping through Equation (18), with $a$ decaying at a rate of $d a / d t \sim-\sqrt{a(t)}$. The solution to this ODE yields a quadratic dependence of $a$ on $t$, so that the time for the orbit to formally shrink from semimajor axis $a$ to zero goes as $\sqrt{a}$. While the orbital evolution halts earlier, as $a$ approaches $q$ (see Fig. 2), when $a \gg q$ we may approximate the time for the orbit to circularize as

$$
\begin{aligned}
\tau_{\text {damp }} & \approx 2 \pi \bar{\rho}\left(G M_{*}\right)^{1 / 2} \frac{\mu_{0} a^{1 / 2} q^{6}}{R_{*}^{6} B_{*}^{2}} \mathcal{F}^{-1} \\
& \approx 160\left[\frac{\bar{\rho}}{5 \mathrm{~g} / \mathrm{cm}^{3}}\right]\left[\frac{M_{*}}{0.5 \mathrm{M}_{\odot}}\right]^{1 / 2}\left[\frac{R_{*}}{0.5 \mathrm{R}_{\odot}}\right]^{-6}\left[\frac{B_{*}}{5 \mathrm{kG}}\right]^{-2}\left[\frac{q}{1 \mathrm{R}_{\odot}}\right]^{6}\left[\frac{a}{5 \mathrm{R}_{\odot}}\right]^{1 / 2} \mathcal{F}^{-1} \mathrm{Myr} ;
\end{aligned}
$$

in the lower equation, the power-law indices apply to an asteroid with an efficiency factor near the maximum, while the numerical reference values are typical of a magnetic $M$ dwarf, as in Equation (20) and Table 1.

Our estimates here are intended to provide a simple scaling relation for Ohmic power dissipation and orbital evolution time scales (e.g., Eq. (23)). Our result is limited to eccentricities above 0.4 in the case of orbits in the equatorial plane, and tend to significantly underestimate the damping time as eccentricity falls below that value. On the other hand, if $i>0$, the damping time is faster since the asteroid encounters stronger magnetic field variations when its motion is not limited to the plane perpendicular to the stellar dipole. Eventually, these orbits circularize and inspiral. Secular perturbation theory offers a way to give a more complete description of these more general scenarios (e.g., Milani et al. 1988). Alternatively, we can use numerical simulations (e.g., Figure 22). Both approaches require an instantaneous force, as in Equation (21) for low Reynolds number.

Unlike the circular orbit case, the efficiency factor does not change significantly as eccentricity damps at fixed $q \ll a$. Another difference is that circular orbits must be inclined relative to the stellar dipole moment for Ohmic heating to occur, whereas eccentricity damping will occur at any orientation relative to the stellar dipole.

The damping time depends strongly on the periastron distance $\left(\tau_{\text {damp }} \sim q^{6}\right)$. Small asteroids $\left(r_{\text {ast }}<1 \mathrm{~km}\right)$ with high tensile strength may be able to survive close to the host star, even when $q$ is much less than a Solar radius (e.g., Kenyon \& Bromley 2017a). Closer to the host star, the magnetic field strength and the efficiency factor for smaller metallic objects are potentially much larger. Orbits for these objects can circularize very quickly compared to the $160 \mathrm{Myr}$ time scale in Equation (23). 


\subsection{The requisite stellar magnetic field}

Our estimates of orbital decay and damping rates (Eqs. 190 and (22)) are appropriate to magnetic red dwarfs. Other stars also provide environments where damping and inspiral may occur. To explore the possibilities, we adopt an optimal asteroid size (Eq. (14)) for orbits near the Roche limit or stellar surface for each of several kinds of stars, listed in Table 1. We then derive the strength of the minimum magnetic field required for an orbital decay time scale of $1 \mathrm{Myr}$, comparing this value with observations.

Table 1: Typical and/or adopted parameters for various stellar hosts

\begin{tabular}{|c|c|c|c|c|c|}
\hline stellar host & $M_{*}$ & $R_{*}$ & $B_{*}$ & $q$ & comment/references \\
\hline red dwarf & $0.5 \mathrm{M}_{\odot}$ & $0.5 \mathrm{R}_{\odot}$ & $5 \mathrm{kG}$ & $1 \mathrm{R}_{\odot}$ & (Morin 2012) \\
\hline Ap star & $2 \mathrm{M}_{\odot}$ & $1.7 \mathrm{R}_{\odot}$ & $30 \mathrm{kG}$ & $5 \mathrm{R}_{\odot}$ & (e.g., Elkin et al. 2010) \\
\hline white dwarf & $0.7 \mathrm{M}_{\odot}$ & $1.4 \mathrm{R}_{\oplus}$ & $10^{8} \mathrm{G}$ & $1 \mathrm{R}_{\odot}$ & Angel et al. 1981) \\
\hline neutron star & $1.4 \mathrm{M}_{\odot}$ & $10 \mathrm{~km}$ & $10^{15} \mathrm{G}$ & $1 \mathrm{R}_{\odot}$ & (Kaspi \& Beloborodov 2017) \\
\hline
\end{tabular}

Figure 3 shows the results. The strongest observed magnetic fields around late-type mainsequence stars $\left(\sim 5 \times 10^{3} \mathrm{G}\right.$; Morin 2012) and white dwarfs $\left(10^{9} \mathrm{G}\right.$; García-Berro et al. 2016) are high enough to cause orbital inspiral on megayear time scales. The field strengths of neutron stars $\left(10^{15} \mathrm{G}\right.$; Kaspi \& Beloborodov 2017) are insufficient. While the magnetic field is strong at the surface of a neutron star, it is comparatively weak near the Roche limit.

To summarize, orbital evolution on a time scale of $1 \mathrm{Myr}$ is possible around some solar-mass stars if the local magnetic field strength near the Roche limit is greater than $\sim 50$ G. For mainsequence stars, this condition means field strengths at the stellar surface of a few kilogauss or more. For white dwarfs, surface fields of at least $10^{8} \mathrm{G}$ are required. Neutron stars, specifically magnetars, seem promising, but the high field strengths at the stellar surface are mundane at orbital distances considered here. We do not give up on neutrons stars, however, as we discuss in $\$ 5$ (see Cordes \& Shannon 2008).

\section{Thermal evolution}

Electromagnetically induced eddy currents in a conducting asteroid produce heat, entirely at the expense of the asteroid's orbital energy. How the thermal energy from Ohmic heating is distributed throughout the asteroid depends on its structure and composition. Furthermore the full thermal evolution of the asteroid must take into account stellar radiation and radiative losses, as well as the heat flux within it. We consider all of these effects in this section. 


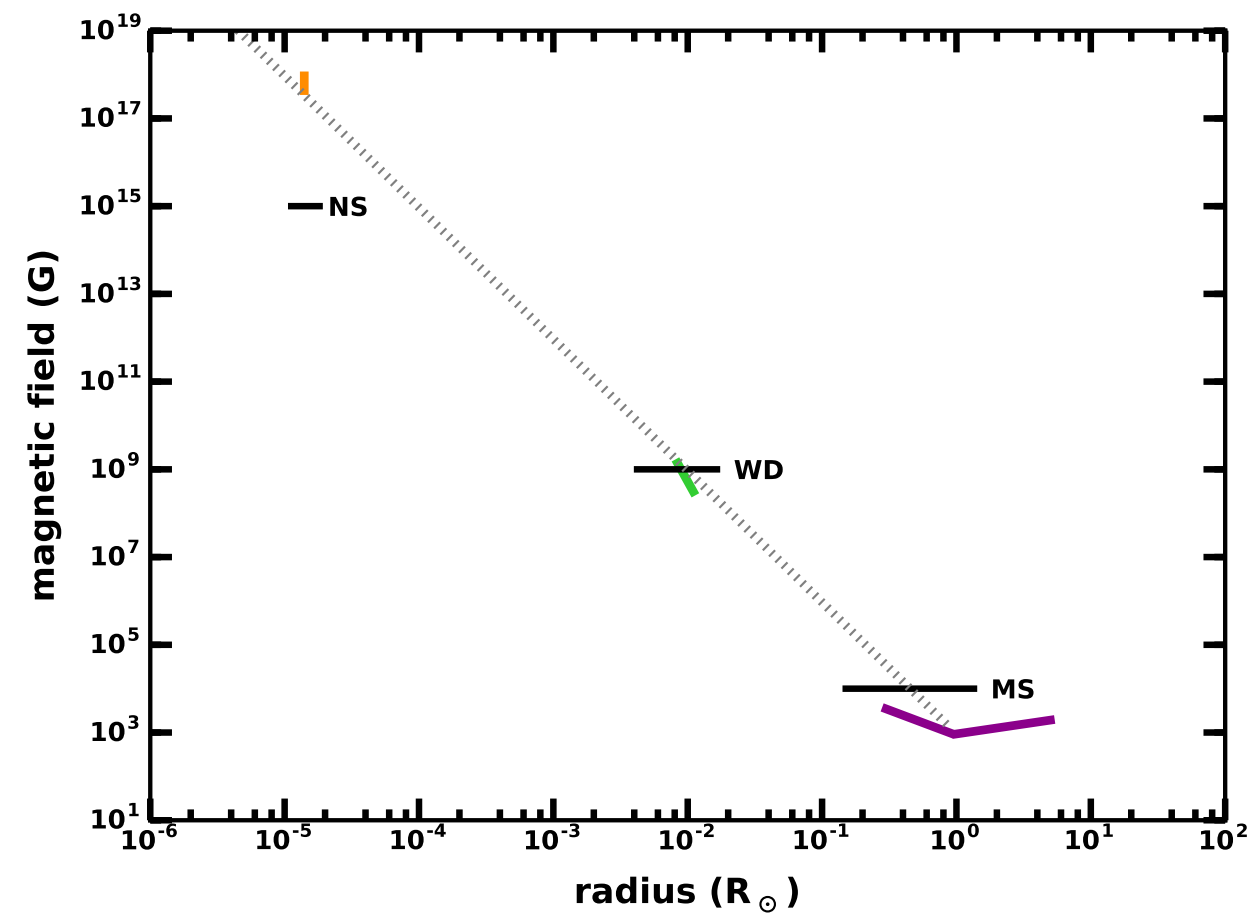

Fig. 3.- Comparison between observed magnetic field strengths and the field strength that is required to cause inspiral of a conducting asteroid within $1 \mathrm{Myr}$, with focus on. neutron stars, white dwarfs, and main-sequence stars. The horizontal lines are the maximum observed magnetic field strengths for each of the three types of stars. The colored line segments are the required field strengths for inspiral. For host radii below $1 \mathrm{R}_{\odot}$, we assume that the asteroid's orbit is at our fiducial Roche limit; for larger stars, the orbital distance is at $1.1 R_{*}$. Because the inspiral rate has some dependence on stellar mass, the required field strengths reflect the mass-radius relation. For neutron stars, we assume masses in the range 1.4-2.5 $\mathrm{M}_{\odot}$, and a fixed stellar radius $(10 \mathrm{~km})$. The dotted reference line below a solar radius shows the required field strength when the mass of the stellar host is fixed at $1 \mathrm{M}_{\odot}$. 
It turns out that thermal evolution time scales for the cases considered here tend to be fast compared to the orbital time scales described in $\$ 3$. Thus, while orbital elements change during thermal evolution (indeed, the latter are driven by the former), we assume that the changes are small and that Ohmic heating rates are constant. Relaxing this assumption generally leads to slightly more rapid thermal evolution than we described here.

To get a sense of the impact of Ohmic heating, we first consider a simple homogeneous body with heat capacity $C_{p}$ and a radius $r_{\text {ast }}$ that is optimal for Ohmic dissipation (Eq. (14). Given some input power from electromagnetic induction (Eq. (5) and neglecting other sources and sinks of energy, the asteroid experiences an average temperature increase at a rate of

$$
\begin{aligned}
\frac{\Delta T}{\Delta t} & =\frac{3\left\langle P_{\mathrm{Ohm}}\right\rangle}{4 \pi C_{p} \bar{\rho} r_{\mathrm{ast}}^{3}} \\
& \approx 10\left[\frac{\bar{\rho}}{5 \mathrm{~g} / \mathrm{cm}^{3}}\right]^{-1}\left[\frac{C_{p}}{0.85 \mathrm{~J} / \mathrm{K} \cdot \mathrm{g}}\right]^{-1}\left[\frac{M_{*}}{0.3 \mathrm{M}_{\odot}}\right]^{1 / 2}\left[\frac{R_{*}}{0.5 \mathrm{R}_{\odot}}\right]^{6}\left[\frac{B_{*}}{5 \mathrm{kG}}\right]^{2}\left[\frac{q}{1 \mathrm{R}_{\odot}}\right]^{-15 / 2} \mathcal{F} \mathrm{K} / \mathrm{yr},
\end{aligned}
$$

where the numerical values for bulk properties are typical of terrestrial material (Elkins-Tanton et al. 2011), while the astrophysical parameters are based on a close-in circular polar orbit around a magnetic red dwarf (Table 1). An asteroid on an eccentric orbit with a similar closest approach and apoastron at $\sim 10 \mathrm{R}_{\odot}(0.05 \mathrm{AU})$ heats up more slowly, at a rate of over ten degrees a century.

As the asteroid heats up, it will also radiate. When a cold asteroid of homogeneous composition has a circular polar orbit, it eventually reaches a surface temperature of

$$
T_{s}=\left(\frac{\left\langle P_{\mathrm{Ohm}}+P_{\mathrm{rad}}\right\rangle}{4 \pi \sigma_{\mathrm{SB}} r_{\mathrm{ast}}^{2}}\right)^{1 / 4} \sim \begin{cases}r_{\mathrm{ast}}^{1 / 4} M_{*}^{1 / 8} R_{*}^{3 / 2} B_{*}^{1 / 2} a^{3 / 4} \mathcal{F}^{1 / 4} & \left(\left\langle P_{\mathrm{Ohm}}\right\rangle \gg\left\langle P_{\mathrm{rad}}\right\rangle\right) \\ T_{*}\left(R_{*} / 2 a\right)^{1 / 2} & \left(\left\langle P_{\mathrm{Ohm}}\right\rangle \ll\left\langle P_{\mathrm{rad}}\right\rangle\right),\end{cases}
$$

where the limiting cases in the rightmost expressions apply when the power from Ohmic heating dominates over the power from stellar heating $\left(P_{\mathrm{rad}}\right)$ and visa versa. Here we assume that the $P_{\mathrm{rad}}$ is the total stellar flux incident on the asteroid's $\pi r_{\text {ast }}^{2}$ cross section (its albedo is negligible) and that the asteroid is a black body radiator. If we neglect stellar radiation, the temperature of an optimal-size asteroid around a red dwarf is

$$
T_{s} \approx\left\{\begin{aligned}
400 \mathrm{~K} & \left(\text { iron } / \text { nickel, } r_{\text {ast }}=3 \mathrm{~km}\right) \\
730 \mathrm{~K} & \left(\text { water, } r_{\text {ast }}=64 \mathrm{~km}\right) \\
1600 \mathrm{~K} & \left(\text { rock, } r_{\text {ast }}=1400 \mathrm{~km}\right)
\end{aligned}\right.
$$

based on electrical conductivities indicated in Equations (15)-(17). Solid objects smaller than the optimal radius radiate interior heat efficiently and are cooler. Although it is harder for larger objects to radiatively cool, the Ohmic heating is also less efficient. With $\mathcal{F} \sim 1 / r_{\text {ast }}$ for $r_{\text {ast }}>r_{\text {opt }}$, larger bodies are also cooler than asteroids of optimal size. In the absence of other heat sources, the listed temperatures are as hot as asteroids get when their conducting cores are exposed.

The values in Equation (27) are also representative of orbits around white dwarfs and Ap stars with parameters listed in Table 1. The highest temperature listed, $1400 \mathrm{~K}$, is comparable to an 
asteroid's equilibrium temperature from heating by stellar radiation from the dwarf stars. At least with regard to energy flow at the asteroid's conducting surface, Ohmic dissipation is only barely competitive with radiative heating, and only for low-luminosity hosts.

\subsection{Heat flow within an asteroid}

In general, Ohmic heating preferentially occurs near the surface of the conducting core. In the low Reynolds number regime (I), the angle-averaged current density in a spherical conductor is linear in distance from the center. With the power density scaling as the square of the current density, the total Ohmic power loss in thin concentric shells with radii $r_{s}$ is proportional to $r_{s}^{4}$. Although eddy currents exist throughout the conductor in this regime, two thirds of the power is deposited in the outer $20 \%$ of the conducting core. At higher Reynolds number where the skin effect is important, all the heating occurs in a thin surface layer; in an iron/nickel core, this layer has a formal depth of less than a centimeter.

As the surface of the conducting core heats up, the thermal energy spreads to neighboring regions, according to the thermal diffusivity of the medium, $\alpha$. For an iron/nickel core, $\alpha=$ $0.5 \mathrm{~cm}^{2} / \mathrm{s}$ (see Elkins-Tanton et al. 2011); for rocky material, the diffusivity is lower, $0.03 \mathrm{~cm}^{2} / \mathrm{s}$ (e.g., Gibert et al. 2003: Šrámek et al. 2012). For any homogeneous, spherical asteroid, the time scale for spreading heat is

$$
\begin{aligned}
\tau_{\text {diff }} & =\frac{r_{\text {ast }}^{2}}{\pi^{2} \alpha} \\
& \approx 580\left[\frac{r_{\text {ast }}}{3 \mathrm{~km}}\right]^{2}\left[\frac{\alpha}{0.5 \mathrm{~cm}^{2} / \mathrm{s}}\right]^{-1} \mathrm{yr} .
\end{aligned}
$$

In the lower equation, numerical values are appropriate for an iron/nickel sphere. Kilometer-size metal asteroids reach thermal equilibrium quickly compared to orbital evolution time scales. Large rocky bodies, with radii of $10^{3} \mathrm{~km}$ or more, have diffusion times exceeding 1 Gyr. Heat transport in large bodies is faster if there are bulk flows or convection, as in the Earth's mantle and water worlds (e.g., Munk 1966).

\subsection{Ohmic heating with a non-conducting mantle}

The thermal history of differentiated solids is more interesting. Asteroids with an iron-rich conducting core and a non-conducting silicate mantle are common (e.g., Elkins-Tanton et al. 2011, Neumann et al. 2012, Scheinberg et al. 2015). With this structure, the mantle traps heat generated in the core, significantly increasing the core temperature from Ohmic heating. 
To assess this effect, we assume that an asteroid is made of a conducting core with radius $r_{c}$, surrounded by a mantle/crust of thickness $\Delta r=\left(r_{\text {ast }}-r_{c}\right)$, where $r_{\text {ast }}$ is the asteroid's full radius. Table 2 shows the bulk properties of the core and the mantle. In this model, Ohmic heating takes place only in the core. While the instantaneous eddy currents from electromagnetic induction within the core have cylindrical symmetry (Eq. (1)), we assume that on average, heating is evenly distributed within concentric spherical shells.

Table 2: Bulk properties of asteroids

\begin{tabular}{|c|c|c|c|}
\hline name & symbol & fiducial value & comment/references \\
\hline \multicolumn{4}{|l|}{ metallic core: } \\
\hline density & $\rho$ & $8 \mathrm{~g} / \mathrm{cm}^{3}$ & (iron/nickel in differentiated bodies) \\
\hline electrical conductivity & $\sigma$ & $1 \times 10^{6} \mathrm{~S} / \mathrm{m}$ & de Koker et al. $(2012)$ \\
\hline relative permeability & $\mu_{\text {rel }}$ & $5 \times 10^{3}$ & (if $T>T_{\text {Curie }} \equiv 10^{3} \mathrm{~K}, 1$ otherwise) \\
\hline thermal diffusivity & $\alpha$ & $0.5-1.0 \mathrm{~cm}^{2} / \mathrm{s}$ & Elkins-Tanton et al. (2011) \\
\hline specific heat & $C_{p}$ & $0.85 \mathrm{~J} / \mathrm{g} \mathrm{K}$ & Bartels \& Grove (1991) \\
\hline solidus/liquidus & - & $1213 / 1233 \mathrm{~K}$ & Formisano et al. $(\overline{2013})$ \\
\hline heat of fusion & - & $250 \mathrm{~J} / \mathrm{g}$ & (appropriate to iron) \\
\hline \multicolumn{4}{|l|}{ rocky mantle/crust: } \\
\hline density & $\rho_{m}$ & $3 \mathrm{~g} / \mathrm{cm}^{3}$ & \\
\hline electrical conductivity & $\sigma_{m}$ & $0.01 \mathrm{~S} / \mathrm{m}$ & Xu et al. (1998) \\
\hline thermal diffusivity & $\alpha_{m}$ & $0.03-1.0 \mathrm{~cm}^{2} / \mathrm{s}$ & Opeil et al. $(2010)$ \\
\hline specific heat & $C_{p, m}$ & $0.80 \mathrm{~J} / \mathrm{g} \mathrm{K}$ & Ghosh \& McSween 1998$)$ \\
\hline solidus/liquidus & - & $1425 / 1850 \mathrm{~K}$ & (Formisano et al. 2013) \\
\hline heat of fusion & - & $400 \mathrm{~J} / \mathrm{g}$ & Ghosh \& McSween (1998, silicates) \\
\hline
\end{tabular}

When the heat flow through the asteroid reaches a steady state, the energy flux through the mantle equals the Ohmic power dissipated in the core. In a simple model where the thermal conductivity of the mantle is constant, the core temperature is

$$
T_{c} \sim \frac{\left\langle P_{\mathrm{Ohm}}\right\rangle f_{m}}{4 \pi \rho_{m} C_{p, m} \alpha_{m} r_{c}}+T_{s}
$$

$\rho_{m}, C_{p, m}$, and $\alpha_{m}$ are the density, specific heat, and thermal diffusivity of the mantle, respectively, and $f_{m}=\Delta r / r_{\text {ast }}$ is the mantle's thickness relative to the full radius. The surface temperature of the asteroid, $T_{s}$ (in Eq. (26)), depends on the stellar radiation, but also on the rate of the heat transport between the core and the surface. On a close-in orbit, the high heating rate and low thermal diffusivity of rock conspire to give formal values of $T_{c}$ that can exceed tens of thousands of degrees even with negligible power input from starlight.

As an example, an asteroid with a $3 \mathrm{~km}$ iron/nickel core on a close-in circular polar orbit around a red dwarf (Table 1) reaches a temperature around $400 \mathrm{~K}$ (Eq. (27)) if the core is exposed. An insulating mantle of only $0.15 \mathrm{~km}$ thickness formally allows the core temperature to reach more 
than 25,000 K. However, as the core is a ferromagnet, it will not reach this temperature. Instead, as the core temperature rises through the Curie point, around $1000 \mathrm{~K}$, the permeability and the efficiency factor for Ohmic heating drop. The core then settles to a steady state with $T_{c}$ near the Curie point, depending on the details of how $\mu_{\text {rel }}$ depends on temperature and the influence of stellar radiation on such a close-in orbit.

If the asteroid had a substantially thicker insulating blanket, then the prediction for the core temperature would lie significantly above the Curie point. With a mantle that is $3 \mathrm{~km}$ thick, the same as the core radius, the formal core temperature at steady state would be over $9000 \mathrm{~K}$. As this example shows, trapping of heat by a crust or mantle allows for high core temperatures in conducting asteroids with a wide range of sizes and composition, even if they are not of optimal size for Ohmic heating.

In a more realistic scenario, the mantle that blankets an asteroid's Ohmic-heated core is dynamic, with regions nearer the core that melt and transport heat efficiently by convection instead of molecular diffusion. Efficient heat flow within the mantle cools the core, transporting energy toward the asteroid's surface where it can be radiated away along with the heat from stellar radiation. We include these effects in the examples we provide next.

\subsection{Thermal evolution of a large asteroid: two examples}

To show how Ohmic heating plays out in an astrophysical context, we choose a large, differentiated asteroid like 4 Vesta, on a close-in, eccentric orbit around a $0.5 \mathrm{M}_{\odot}$ red dwarf with an effective temperature of $3500 \mathrm{~K}$ (Table 1). The asteroid's orbit has a periastron distance of $1 \mathrm{R}_{\odot}$ and semimajor axis of $5 \mathrm{R}_{\odot}$, about $0.005 \mathrm{AU}$ and $0.025 \mathrm{AU}$, respectively. The asteroid's physical radius is $r_{\text {ast }}=250 \mathrm{~km}$, and its metallic core radius $r_{c}$ is half that size. Table 2 lists bulk properties. We assume that the asteroid acquires its orbit in a cold state with a ferromagnetic core, and that it heats up through the Curie temperature and melting points, maintaining a high electrical conductivity in the core and relatively low conductivity elsewhere.

We estimate the thermal evolution from the 1-D spherical thermal diffusion equation, solving it with a finite difference code, as in Hevey \& Sanders (2006). At the outer boundary, the surface temperature of the asteroid is set to balance input of thermal energy flow from the interior of the asteroid and from starlight of the host with loss from blackbody radiation. At the inner boundary, the temperature gradient is zero. Energy input from eddy currents depends on the magnetic Reynolds number. For a ferromagnetic iron/nickel core, $R_{m}$ is high (regime III); energy is deposited in a single finite-difference bin. If $R_{m}$ is low (regime I), the power is distributed as across bins as $r^{4}$. We set time steps using the Courant condition, and also iteratively smooth the sharp discontinuity in the thermal conductivity between the core and the mantle with a box car 
function as needed for numerical stability.

The code tracks the phase of matter and accounts for heat of fusion as solids go through melting, similar to the algorithm of Elkins-Tanton et al. (2011). The transition across the Curie point is assumed to be sharp, with $\mu_{\text {rel }}$ modeled as a step function. When the temperature in a bin exceeds the melting point, we increase the thermal diffusivity linearly with temperature between solidus and liquidus from the thermodynamic value to $\alpha=1.0 \mathrm{~cm}^{2} / \mathrm{s}$ as a way to mimic convective heat flow (Hevey \& Sanders 2006; Sahijpal et al. 2007), although this choice may underestimate the effect (.e.g Neumann et al. 2014). We assume that there is no mixing between a liquid core and a liquid mantle.

Figure 4illustrates the thermal evolution of the Vesta-like asteroid on an eccentric orbit around its red dwarf host. The plot shows temperature as a function of depth from the asteroid's surface and how it changes with time. We assume that the asteroid is initially cold; as indicated in the Figure, the core temperature rapidly heats up beyond our adopted Curie point of $1000 \mathrm{~K}$. The mantle starts melting after about $15 \mathrm{Myr}$, and forms a region of partial melt that extends to a crust of about $20 \mathrm{~km}$ thick. Stellar radiation also contributes, helping to maintain a surface temperature above $800 \mathrm{~K}$, close to the equilibrium temperature for the asteroid's eccentric orbit.

We explore variations on the scenario depicted in Figure 4. If the Vesta-like asteroid were to achieve a circular orbit at that same perihelion, the core could experience runaway Ohmic heating. Figure 5 provides an illustration where $T_{c}$ grows well above $10,000 \mathrm{~K}$. While the state of matter at these temperatures is unclear to us, the configuration does not seem stable. The role of stellar radiation in the thermal evolution is to quickly bring the surface temperature to just below liquidus. The asteroid thus becomes a lava sphere, with an extraordinary amount of thermal energy building up in its core.

The role of stellar radiation is notable in both of the above examples. For the asteroid on an eccentric orbit, for which the equilibrium temperature is cool (about $780 \mathrm{~K}$ ), stellar heating causes the convective melt zone to extend radially outward, thereby increasing the efficiency of heat transport from the core. Although starlight is a significant part of the energy budget, it has only modest impact on the core temperature. For the asteroid on a close-in orbit, starlight alone can heat the surface rock almost to liquidus. Furthermore, because the mantle is almost fully melted a homogeneous medium in our model - the equilibrium temperature adds to the steady-state core temperature (Eq. (30)). However, Ohmic heating is so strong that the contribution from stellar radiation is not important.

When the core temperatures are above solidus, volcanism is a possible outcome (e.g., Wilson \& Keil 1991; Taylor et al. 1993). A modest amount of volatile elements in the mantle could drive explosive eruptions (Wilson \& Keil 1991), providing obscuring clouds of dust and a mechanism for metals from the asteroid to pollute the stellar surface. In an evolved asteroid, it is unclear how 


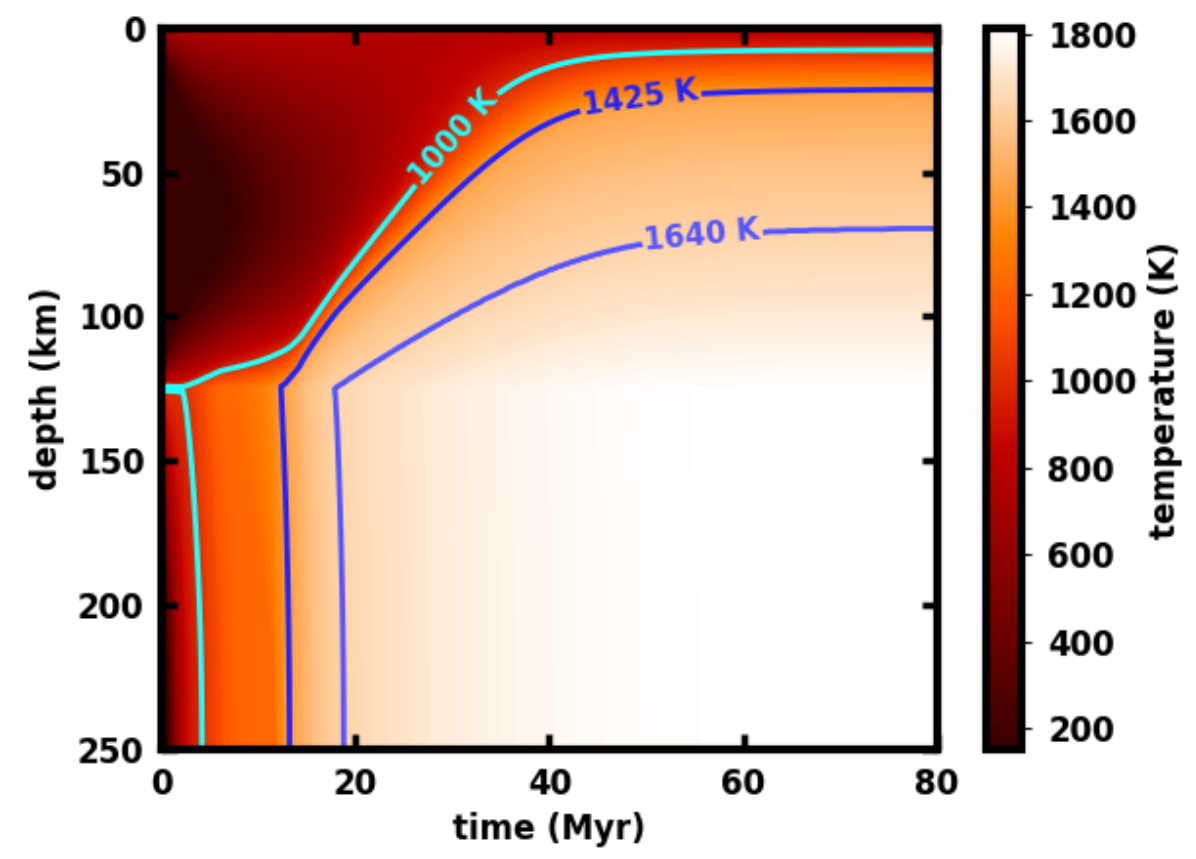

Fig. 4. - The thermal evolution of a $250 \mathrm{~km}$ differentiated asteroid with an iron/nickel core and a rocky mantle on an eccentric orbit around a red dwarf. This density plot shows the temperature as a function of depth from the surface (vertical axis), and time (horizontal axis). The "isotherm" at $1425 \mathrm{~K}$ corresponds to solidus for the mantle material; the contour at $1625 \mathrm{~K}$ is the $50 \%$ melt temperature. The cyan contour indicates our adopted Curie temperature, applicable to an $\mathrm{Fe} / \mathrm{Ni}$ core. Stellar radiation also contributes to the overall thermal evolution. Its impact is evident from the increase in temperature near the asteroid's surface over the first $\sim 10 \mathrm{Myr}$. 


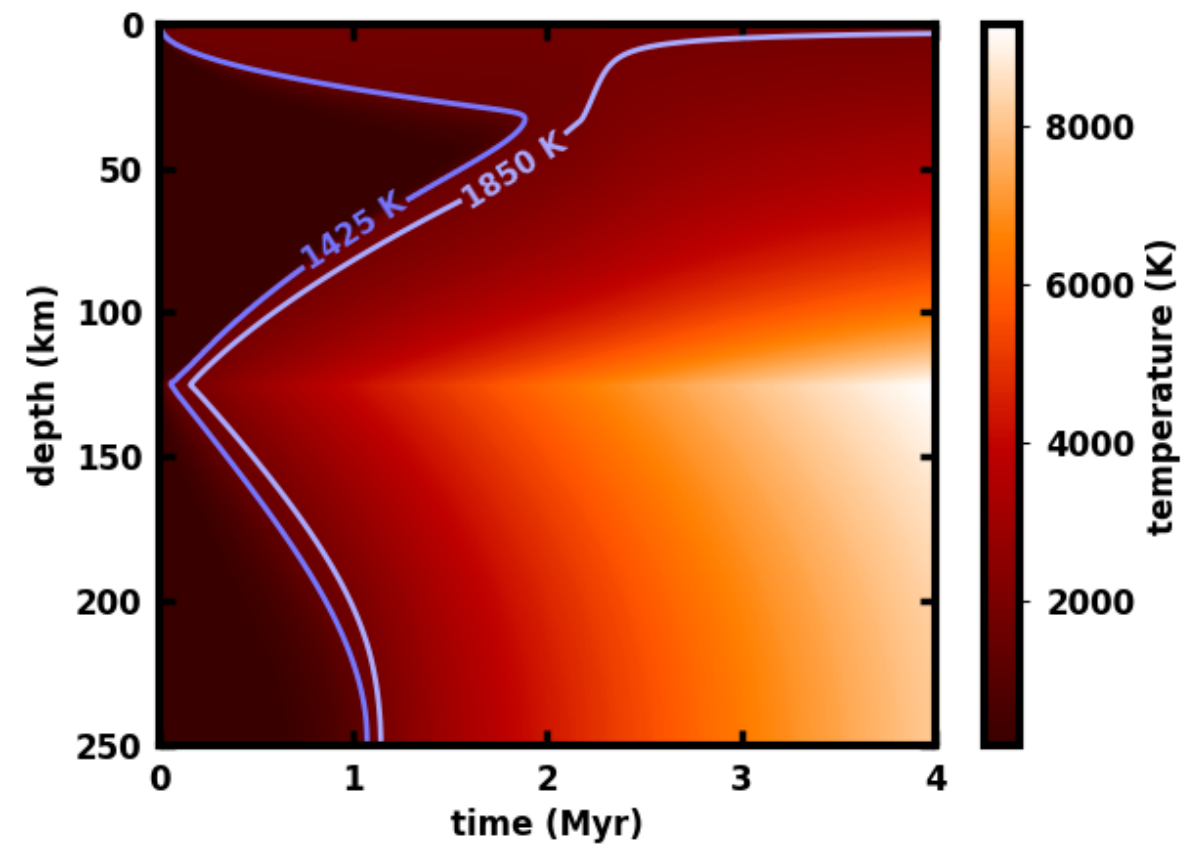

Fig. 5. - The thermal evolution of a $250 \mathrm{~km}$ differentiated asteroid with an iron/nickel core and a rocky mantle on a close-in circular orbit $(0.005 \mathrm{AU})$ around a red dwarf. This density plot shows the temperature as a function of depth and time, as in Figure 4. Here, we show isothermal contours for the solidus $(1425 \mathrm{~K})$ and liquidus of the mantle $(1850 \mathrm{~K})$. In this case, the Ohmic heating rate is high and the core temperature rises considerably above liquidus. Because the asteroid is close its stellar host, its equilibrium temperature with is close to liquidus. Thus, the asteroid is nearly completely melted except for a thin semi-solid crust. 
much volatile material might have survived earlier epochs of heating or whether the hard crust is too thick for liquid to break through to the surface (e.g., Elkins-Tanton et al. 2011). If crust thickness is the main issue, the two scenarios depicted in Figures 4 (cool, thick solid crust) and 5 (hot, essentially a complete melt) likely bracket conditions for volcanism.

To summarize this section, the thermal history of asteroids experiencing Ohmic heating is rich and includes the formation of a deep magma ocean and runaway heating of the core. We caution that the idealization of a two component (conducting+non-conducting) asteroid may be naive. For example, in the $250 \mathrm{~km}$ differentiated asteroid in Figures 4 and 5, we ignore that the mantle becomes a magma ocean with conductivity that might be significant. If $\sigma=1 \mathrm{~S} / \mathrm{m}$ or more (e.g., Gaillard \& Marziano 2005) this ocean can inductively heat even more efficiently than the metal core. Other interesting physics may be missed by our use of a 1-D thermal diffusion solver, as when an asteroid is tidally locked to the stellar host. Temperatures would build up to higher levels and in more localized regions than in our calculations.

\section{Discussion}

Guided by the estimates provided in the previous sections, we examine how electromagnetic induction and Ohmic heating might play out for several types of conducting bodies (\$5.1) around different kinds of stellar hosts $(\$ 5.2)$.

\subsection{The orbiting bodies}

\subsubsection{Cold iron/nickel cores}

On an orbit with periastron of $1 \mathrm{R}_{\odot}$ around a solar-mass star, the optimal size of a cold, ferromagnetic iron-nickel core is about $3 \mathrm{~km}$. This core is capable of eccentricity damping on time scales of hundreds of millions of years around the stellar hosts considered here (Table 1). The damping time could be significantly smaller, if those hosts have stronger magnetic fields or if the periastron distance is inside the nominal Roche radius (Eq. (22)). Once settled close to the host star, stellar radiation alone causes the asteroid's core temperature to rise above the Curie point. Then, Ohmic heating no longer operates efficiently (the core is in regime III, $R_{m} \gg \mu_{\text {rel }}^{2} \sim 1$ ). Heat input and orbital evolution slow.

If a heated core manages to melt the surrounding mantle, it is possible that eddy currents form in the magma ocean instead. If the magma conductivity were $\sim 1 \mathrm{~S} / \mathrm{m}$, then this scenario would lead to efficient Ohmic heating and orbit evolution if the surface of the ocean were at a radius of roughly $150 \mathrm{~km}$. A smaller size would be optimal if the asteroid were on an orbit were closer than 
a Solar radius to its host star.

\subsubsection{Hot metallic asteroids or meteors}

A cold, small iron/nickel asteroid, $r_{\text {ast }} \lesssim 1 \mathrm{~km}$ will orbitally damp slowly, likely not on interesting time scales. However, if the asteroid is on a close-in orbit and its temperature rises above the Curie point, then the efficiency of Ohmic heating increases. The optimal radius of an orbiting metallic paramagnetic sphere is roughly $150 \mathrm{~m}$. In this case, heating is possible, but cooling is efficient, too. The meteor will inspiral through the Roche limit to be tidally shredded and accreted by the star.

\subsubsection{Water worlds}

At an optimal size of $\sim 70 \mathrm{~km}$, a water world is capable of circularizing to a close-in orbit around a magnetic host star. Once on a tight orbit, however, the temperature of a water world may easily rise above the boiling point from Ohmic heating or stellar radiation. The water then evaporates. Simple estimates suggest that a water world receives enough energy from the light of a red dwarf or white dwarf to vaporize a kilometer-deep layer per year. A water world will not last long on a close-in orbit.

\subsubsection{Rocky asteroids and dwarf planets}

With electrical conductivity in the range of $10^{-4} \mathrm{~S} / \mathrm{m}$ to $1 \mathrm{~S} / \mathrm{m}$, rocky bodies have an optimal radius between $\sim 100 \mathrm{~km}$ (high $\sigma$ ) and $\sim 10,000 \mathrm{~km}$ (low $\sigma$ ). An object with the intermediate value of $1400 \mathrm{~km}(\sigma=0.01 \mathrm{~S} / \mathrm{m})$ could qualify as a dwarf planet. Despite its size, it can experience orbital damping on a time scale of $\sim 100 \mathrm{Myr}$, and inspiral within $\sim 1 \mathrm{Myr}$. If the planet had an insulating crust with low thermal diffusivity compared to the bulk, then the interior could heat beyond the melting point on Myr-time scales, comparable to orbital evolution times. In Vesta-size bodies with radii below optimal for Ohmic heating, a melted interior could raise the efficiency $\mathcal{F}$, since the optimal radius of a magma ocean is around $150 \mathrm{~km}$. 


\subsection{The stellar hosts}

\subsubsection{Red dwarfs}

Because of their combination of strong surface magnetic field, stellar radius and mass, magnetic red dwarfs provide a venue for Ohmic heating of asteroids by electromagnetic induction. Numerical values for time scales for orbital damping, inspiral, and heating given above are tuned for typical magnetized K and M stars; These processes can be amplified or accelerated by moving the periastron distance $q$ to well within the nominal Roche limit (Eq. $(13)$ ), or by choosing a host with a stronger surface magnetic field strength and/or larger radius. For example, moving $q$ from $1 \mathrm{R}_{\odot}$ to $0.8 \mathrm{R}_{\odot}$ shortens the damping time by a factor of four and the inspiral time by a factor of five. Increasing the magnetic field strength from $5 \mathrm{kG}$ by $50 \%$ (near the upper limit of observed values Johns-Krull et al. 2009) decreases these time scales by another factor of two.

\subsubsection{Other main-sequence or pre-main-sequence stars}

T Tauri stars and magnetic Ap stars have comparable stellar radii $\left(\sim 2 \mathrm{R}_{\odot}\right)$ and strong surface magnetic field strengths (up to 1-30 kG). Inspiral and eccentricity damping times for these objects are short compared to red dwarfs because of their greater magnetic field strength and the faster orbital speeds at comparable distance.

Around HD 215441 (Babcock's (Ap) star; Babcock 1960) with a field strength of $34 \mathrm{kG}$, an optimal-size asteroid or dwarf planet with periastron $q=5 \mathrm{R}_{\odot}$ (about $0.02 \mathrm{AU}$ ) and apoastron of five times that distance has a damping time of about $190 \mathrm{Myr}$. Moving the periastron distance inward to $3 \mathrm{R}_{\odot}$ reduces the damping time to under $10 \mathrm{Myr}$.

T Tauri stars have weaker fields and make their transition to the main sequence quickly compared with the eccentricity damping time scale. Nonetheless, inspiral on a close-in orbit is also possible around these stars.

\subsubsection{White dwarfs}

Of the $\sim 10 \%$ of white dwarfs that are magnetic, roughly half have field strengths above our fiducial value of $10^{7}$ Gauss (Ferrario et al. 2015, Fig. 8 therein). About a fifth of these high fieldstrength stars - about $1 \%$ of all white dwarfs — have $B_{*}=10^{8}$ Gauss or higher. At this field strength, we expect similar damping, inspiral and heating time scales as for red dwarfs. For the rare objects with $\sim 10^{9} \mathrm{G}$ fields, the time scales all drop by a factor of 100 . With the fiducial stellar parameters in Table 1, based on WD 1145+017 (Veras et al. 2017), the eccentricity damping 
time of an optimal-size asteroid is under $10 \mathrm{Myr}$ if periastron is near the nominal Roche limit and apoastron is within about $0.1 \mathrm{AU}$.

Contributing to electromagnetic induction around a white dwarf is the magnetic flux variation when the magnetic dipole moment is not aligned with the star's spin axis. Magnetic white dwarfs have a rotation period that ranges from under an hour to over several days, with an average of just under one day (Kawka et al. 2007; Brinkworth et al. 2013; Kawaler 2015). For comparison, the orbital period at $1 \mathrm{R}_{\odot}$ around a $0.7 \mathrm{M}_{\odot}$ star is about 3.3 hours. While there is a tendency for the stars with the stronger fields to have rotation periods that are much longer than average (Ferrario \& Wickramasinghe 2005), stellar rotation may impact Ohmic heating of bodies around it.

How "spin-orbit coupling" plays out depends on the alignment between the spin and the dipole, the rotation rate, and the configuration of the asteroid's orbit. In some scenarios (slow rotation or perfect dipole-spin alignment) stellar rotation has little impact. In other scenarios, Ohmic heating is suppressed when the asteroid's orbit corotates with a misaligned magnetic dipole. When the stellar rotation is fast compared to the orbital angular speed, or the orbit counterrotates relative to a spinning dipole, Ohmic heating is magnified. We consider this possibility separately for neutron stars.

\subsubsection{Neutron stars}

With radius of about $10 \mathrm{~km}$, a mass of $1.4 \mathrm{M}_{\odot}$, and a magnetic field strength of $10^{15} \mathrm{G}$ at the stellar surface, a neutron star has extraordinary potential for Ohmic heating. However, the magnetic field strength is less than $5 \mathrm{G}$ near the Roche limit, which is an order of magnitude weaker than around a dwarf star at comparable distance. Electromagnetic induction can still be important for close-in asteroids, but the origin of magnetic flux variation is stellar rotation, not orbital motion (Cordes \& Shannon 2008; Kotera et al. 2016). Thus we focus only on the thermal evolution of the orbiting body.

Neutron stars have a range of spin rates and magnetic field strengths. Millisecond pulsars have frequencies of $f=\omega / 2 \pi \sim 10-1000 \mathrm{~s}^{-1}$ (the pulsar PSR J1748-2446ad has the fastest spin rate, with $f=716 \mathrm{~Hz}$; Hessels et al. 2006) and magnetic field strengths around $10^{8} \mathrm{G}$ (e.g., Mukherjee et al. 2015). Magnetars have dipole fields as strong as $10^{15} \mathrm{G}$ and spin rates of seconds (see Kaspi \& Beloborodov 2017, and references therein). Since efficient Ohmic heating scales as $B^{2} \omega$ (Eq. (7) $)^{3}$, we focus on magnetars, preferring strong field strengths to high spin rates. Although the magnetic dipole and angular momentum are roughly aligned in some magnetars, thereby diminishing the

\footnotetext{
${ }^{3}$ Unlike Kotera et al. (2016), we ignore electromagnetic scattering, treating $\epsilon \omega \ll \sigma$. For the conductivities discussed here, and for observed neutron star spin rates, this assumption remains valid.
} 
amplitude of field oscillations (e.g., Vogel et al. 2014; An et al. 2015), magnetars still are the most promising class of neutron stars for electromagnetic induction and Ohmic heating.

For orbits at $1 \mathrm{R}_{\odot}$, near the nominal Roche limit around a magnetar, and for a stellar rotation frequency of $f=1 \mathrm{~Hz}$, we apply Eq. (10) to obtain the optimal radius of an induction-heated asteroid. When the asteroid is composed of non-magnetic material, we find that

$$
r_{\mathrm{opt}} \sim 1.2\left[\frac{\sigma}{1 \mathrm{~S} / \mathrm{m}}\right]^{-1 / 2}\left[\frac{f}{1 \mathrm{~Hz}}\right]^{-1 / 2} \mathrm{~km} \quad\left(\mu_{\mathrm{rel}} \approx 1\right) .
$$

From this expression, rocky bodies with $\sigma=0.01 \mathrm{~S} / \mathrm{m}$ have an optimal radius of $12 \mathrm{~km}$, while

asteroids with an iron/nickel core heated above the Curie point, $r_{\text {opt }}$ is about $1.2 \mathrm{~m}$. In a separate calculation, we estimate that a cold, ferromagnetic asteroid has an optimal radius of just over $25 \mathrm{~m}$.

The average heating rate in the oscillating magnetic field is

$$
\frac{d T}{d t} \sim 0.9\left[\frac{\bar{\rho}}{5 \mathrm{~g} / \mathrm{cm}^{3}}\right]^{-1}\left[\frac{C_{p}}{0.85 \mathrm{~J} / \mathrm{K} \cdot \mathrm{g}}\right]^{-1}\left[\frac{B_{*}}{10^{15}}\right]^{2}\left[\frac{q}{1 \mathrm{R}_{\odot}}\right]^{-6} \mathcal{F} \mathrm{K} / \mathrm{yr} .
$$

At this rate, a $12 \mathrm{~km}$ rocky body will reach a steady state around $\sim 200 \mathrm{~K}$ in the absence of other energy sources. Larger bodies, with less efficient radiative cooling, reach higher temperatures. A crust with low thermal diffusivity allows for runaway heating and melting in the interior of bodies of all sizes.

\subsection{Observational outcomes}

Given the range of asteroid size, composition and stellar host for which Ohmic heating may be important, we consider a set of possible observational consequences of the phenomenon. We begin with the most promising one.

\subsubsection{Pollution of white dwarf atmospheres}

Sinking times for metals at the surface of a white dwarf is so rapid that no metals should persist in the stellar atmosphere (Dupuis et al. 1992; Althaus \& Benvenuto 2000; Koester 2009). Yet O, $\mathrm{Mg}, \mathrm{Al}, \mathrm{Si}, \mathrm{Ca}$, and Fe are abundant in about a third of all DA and DB stars (e.g., Zuckerman \& Reid 1998; Zuckerman et al. 2010; Gänsicke et al.|2012; Koester et al. 2014; Kepler et al. 2015, 2016, Farihi (2016). There must be a reservoir of external material available to these stars, presumably of planetary origin, delivering mass at an average rate of $10^{5} \mathrm{~g} / \mathrm{s}$ to $10^{10} \mathrm{~g} / \mathrm{s}$ (e.g., Farihi 2016).

Ohmic heating can result in the delivery of metals to the atmosphere of a white dwarf. A small metallic or metal-rich asteroid, scattered onto an orbit within a Solar radius of the stellar host, 
can damp and inspiral to be tidally shredded within its Roche radius. At a periastron distance of ten times the stellar radius, or about $0.1 \mathrm{R}_{\odot}$, a hot, $10 \mathrm{~m}$ metallic asteroid would be near both its Roche limit and the optimal radius $r_{\mathrm{opt}}$. The damping time then is thousands of years for orbits with apoastron at $10 \mathrm{R}_{\odot}$ around a host with a surface magnetic field of strength of $10^{8} \mathrm{G}$. Inspiral is even faster. After tidal shredding, the next steps include evaporation and gas accretion (e.g, Jura 2003, 2008; Debes et al. 2012; Veras et al. 2014; Kenyon \& Bromley 2017b).

Similarly, kilometer-size rocky bodies would be near both the optimal radii and their Roche limit at about a half of a Solar radius (Kenyon \& Bromley 2017a, Fig. 1 therein). Damping time scales from $10 \mathrm{R}_{\odot}$ exceed $1 \mathrm{Myr}$, but inspiral times are only thousands of years around a star with $B_{*}=10^{8} \mathrm{G}$. Our prediction is thus that electromagnetic induction can accelerate the flux of planetary material orbiting close to magnetic stars as compared with their non-magnetic counterparts.

\subsubsection{Dust from explosive volcanism}

Large asteroids or dwarf planets, if near optimal size for Ohmic heating, can experience interior melting (Figures 5 and 4 ) opening up the possibility of volcanism. Volcanic eruptions can produce copious amounts of gas and dusty solids; eruptions on Jupiter's moon Io, driven by heating from tidal stresses (Lainey et al. 2009), contain as much as $10^{9} \mathrm{~g}$ in submicron-size dust (Krüger et al. 2003 Geissler \& McMillan 2008). Terrestrial volcanos can eject thousands to millions of cubic kilometers of material, or over $10^{18} \mathrm{~g}$ if in the form of solids.

The numerous outstanding issues for volcanism include the amount and composition of ejected material, the number of eruption sites, the frequency of eruptions, and the ejecta speed relative to the asteroid's escape velocity. If parameters are favorable for the release of volcanic dust onto close-in orbits, a consequence could be short-lived occultation of the stellar host. By ejecting $10^{14} \mathrm{~g}$ of micron-size particles an eruption can temporarily dim the stellar host (see Kenyon \& Bromley 2005, Eq. 9 therein).

A second consequence of volcanic dust production is the delivery of metals to the stellar host. With $\sim 10^{9} \mathrm{~g}$ of material per event, daily eruptions are needed to account for the observed metal accretion rates in white dwarfs. An annual explosive supervolcano could provide a similar metal pollution rate. 


\subsubsection{Exploding asteroids?}

Ohmic heating of an asteroid with a modest-size conducting core $\left(r_{c} \sim 1 \mathrm{~km}\right)$ and an insulating crust can lead to melting of its interior. If this transition happens near the Roche limit, the loss of material strength allows tidal forces to overwhelm the asteroid, rapidly liberating $10^{15} \mathrm{~g}$ of debris. We speculate that the observational signature would be similar to a catastrophic collision between two asteroids (Kenyon \& Bromley 2005). The distinguishing feature of a tidally shredded asteroid is that the dynamical time corresponding to the debris cloud is fast - hours, not days or months - because of the close-in orbital distance.

As a result of the high temperature build-up in large asteroids $\left(r_{\text {ast }} \gtrsim 10 \mathrm{~km}\right.$, as in Fig. 5), there is the possibility that very hot core material might mix with a cooler, volatile-rich mantle or thick crust. Even a small molar fraction of volatiles (e.g., 1\%), if heated rapidly to $\mathrm{O}\left(10^{3}\right) \mathrm{K}$, could cause a disruptive expansion of gas within the asteroid. The resulting explosive event could be as spectacular as it is speculative.

\subsection{Where to look: Candidates for Ohmic heating}

- High-field magnetic white dwarfs, analogous to WD 1145+017. The handful of white dwarfs with field strengths above $10^{8} \mathrm{G}$ offers the most promising environments for Ohmic heating by electromagnetic induction (e.g., Briggs et al. 2015, Table 9 therein). Observables would be atmospheric pollution by planetary material and, possibly, occultation by dust. The nonmagnetic star WD 1145+017 shows compelling evidence for both phenomena, likely resulting from the tidal disruption of an asteroid near its Roche limit (see Farihi et al. 2018, and references therein). It illustrates the promise of observing close-in asteroids as well as the challenge of disentangling the effects of Ohmic heating from tidal break-up.

- Magnetic main-sequence stars. Ohmic heating is possible around both magnetic red dwarfs and early-type stars. An induction-heated asteroid or dwarf planet that has volcanic activity could produce clouds of obscuring dust. This scenario might be consistent with the deep enigmatic occultations of F star KIC 8462852 (Boyajian et al. 2016). It could also explain the apparent disintegration of small, ultra-fast period planets around red dwarfs, including the "super-Mercury" Kepler-1520 b (aka KIC 12557548; Rappaport et al. 2012) and K2-22b (Sanchis-Ojeda et al. 2015), which are both orbiting at a distance of about $3 \mathrm{R}_{\odot}$ around their hosts.

- Pre-main-sequence stars. The environment around a T Tauri star can be messy, with a vigorous stellar wind, accretion flows, and strong magnetic fields. Induction heating of an asteroid that relies on current through the circumstellar plasma (e.g., Sonett et al. 1970) could be supplemented by Ohmic heating driven by changing magnetic flux if the orbit passes 
close to the stellar surface. There is now evidence of planetary material on close-in orbits about some young stars, as reported by Stauffer et al. (2018). Phased light curves of these sources show deep "scallop shell" undulations with periods of hours, suggestive of close-in circumstellar gas and dust. The light-curve patterns can persist for months, with occasional abrupt changes after flare outbursts. While the connection between these features and Ohmic heating of an asteroid is not clear, they suggest that planetary material can get close to the stellar surface.

- Magnetars. A large asteroid that is induction-heated to the point where it can sustain volcanic activity could result in episodic bursts of radiation from the host. Volcanic ejecta transferred from the asteroid to the neutron star surface will become relativistic when it impacts the surface. We do not speculate further except to note that the rest energy in single volcanic eruption of $10^{9} \mathrm{~g}$ is over $10^{29} \mathrm{erg}$.

\section{Conclusion}

Here, we describe how the orbits of conducting bodies with radii in the range of $\sim 1 \mathrm{~m}$ to over $1000 \mathrm{~km}$ ("asteroids") are modified by electromagnetic induction as they plow through the magnetic field of a stellar host. We focus on objects of rocky or metallic composition, based on simple assumptions: all material is homogeneous and isotropic, with linear response to electromagnetic fields. Our choices of density, electrical conductivity, permeability, thermal diffusivity and specific heat are representative of small bodies in the solar system. We use analytical solutions for the current density that depend on the magnetic Reynolds number and permeability to track Ohmic heating, and a 1-D finite difference code to estimate heat flow within an asteroid.

Our analysis demonstrates that orbital damping and inspiral from Ohmic dissipation is possible if the perihelion distance is near the Roche limit $\left(\sim 1 \mathrm{R}_{\odot}\right)$ around magnetic stars with typical field strengths (Table 1). The time scales for these processes are around 1 Myr to 1 Gyr. Damping and inspiral times can be much shorter if periastron distances that are well inside the nominal Roche limit can be sustained, or if the magnetic dipole field strength were increased. For example, the damping time of an orbit with apoastron at $0.1 \mathrm{AU}$ around a white dwarf falls to about 10,000 years if the magnetic field strength is at the upper end of the observed range $\left(10^{9} \mathrm{G}\right)$, and periastron is at $0.3 \mathrm{R}_{\odot}$. The inspiral time from $0.3 \mathrm{R}_{\odot}$ is a few decades.

Ohmic heating occurs on even faster time scales. When placed on an orbit around a magnetic star that generates electromagnetically induced eddy currents, an asteroid or dwarf planet plausibly heats at rates as high as a few Kelvin per year. Exposed conducting cores of $10^{3} \mathrm{~km}$ or less reach a steady state at an uninteresting temperature (e.g., below the melting point, or less than the equilibrium temperature from radiative heating). A crust or mantle that traps heat causes core temperature to formally rise well above the melting point. 
Our analysis is limited in several ways. First, we consider only the magnetic dipole field of the host star. Stellar magnetic fields may be more complicated (e.g., Putney \& Jordan 1995; Fendt \& Dravins 2000; Wickramasinghe 2001, for white dwarfs) and may alter the flux variations experienced by asteroids on close-in orbits. The magnetic field may also have a component that arises in the circumstellar environment. The magneto-rotational instability within a protoplanetary accretion disk may support field strengths of $100 \mathrm{G}$ or more at distances of a few solar radii (e.g., Mohanty et al. 2018). Second, we do not consider the effect of the electric field induced by the orbital motion of an asteroid through the (approximately uniform) local magnetic field, which may be important in the presence of a plasma or stellar wind (Sonett et al. 1970, see also Mottez \& Heyvaerts 2011 for a study that also includes the back-reaction of the plasma). Finally, we neglect the general effect of local field gradients, including the force on the induced magnetic dipole of a conducting asteroid by the gradient in the stellar field (e.g., Ray et al. 2018). Our focus here is just on phenomena directly tied to the loss of orbital energy.

Despite these limitations, the results presented here show possibilities for detecting Ohmic heating of asteroids and dwarf planets. The effect is most likely to occur in the most extreme cases of close-in orbits and high magnetic field strengths. Promising environments are Ap stars, magnetic red dwarfs, and magnetic white dwarfs. Despite very strong surface fields, orbit-powered Ohmic heating is not effective around neutron stars. Instead, the spinning magnetic dipole moment of a magnetar is substantially more effective as a generator of induced currents and Ohmic heat Cordes \& Shannon 2008; Kotera et al. 2016).

The primary connections of Ohmic heating to observations include orbital inspiral of a conducting asteroid through the Roche limit, delivering a large reservoir of metals to the immediate vicinity of the stellar host. We are encouraged by recent discoveries of ultra-short period planets as a sign that such close-in orbits are achievable (Sanchis-Ojeda et al. 2014b). If an asteroid or dwarf planet has an insulating crust or mantle, then Ohmic heating can raise the temperature of core material high enough to potentially cause volcanic eruptions or large-scale explosions even before tidal break-up. Such events could deliver the elements observed in the atmospheres of metallic white dwarfs, and might generate enough dust to dim main-sequence stars. Around a neutron star, a large volcanic eruption could send ejecta into the deep, relativistic gravitational well, beaming news of the event to viewers at vast distances.

We are grateful to M. Geller, C. Boehme, O. Starykh, C. Williams, and Z. Zheng for comments and helpful discussions. We thank an anonymous referee for providing guidance that significantly improved our manuscript. We also thank J. Nagel for key advice on the problem of induced eddy currents. NASA provided essential support for this program through a generous allotment of computer time on the NCCS 'discover' cluster and Outer Planets Program grant NNX11AM37G. 


\section{REFERENCES}

Alonso, R., Rappaport, S., Deeg, H. J., \& Palle, E. 2016, A\&A, 589, L6

Althaus, L. G., \& Benvenuto, O. G. 2000, MNRAS, 317, 952

An, H., Archibald, R. F., Hascoët, R., et al. 2015, ApJ, 807, 93

Angel, J. R. P., Borra, E. F., \& Landstreet, J. D. 1981, ApJS, 45, 457

Babcock, H. W. 1960, ApJ, 132, 521

Bartels, K. S., \& Grove, T. L. 1991, in Lunar and Planetary Science Conference Proceedings, Vol. 21, Lunar and Planetary Science Conference Proceedings, ed. G. Ryder \& V. L. Sharpton, 351365

Batygin, K., Stevenson, D. J., \& Bodenheimer, P. H. 2011, ApJ, 738, 1

Bidinosti, C., Chapple, E., \& Hayden, M. 2007, Concepts in Magnetic Resonance Part B: Magnetic Resonance Engineering, 31B, 191

Bisnovatyi-Kogan, G. S., \& Lovelace, R. V. E. 1997, ApJ, 486, L43

Boyajian, T. S., LaCourse, D. M., Rappaport, S. A., et al. 2016, MNRAS, 457, 3988

Briggs, G. P., Ferrario, L., Tout, C. A., Wickramasinghe, D. T., \& Hurley, J. R. 2015, MNRAS, 447,1713

Brinkworth, C. S., Burleigh, M. R., Lawrie, K., Marsh, T. R., \& Knigge, C. 2013, ApJ, 773, 47

Bromley, B. C., \& Kenyon, S. J. 2011, ApJ, 731, 101

Chatterjee, S., Ford, E. B., Matsumura, S., \& Rasio, F. A. 2008, ApJ, 686, 580

Cordes, J. M., \& Shannon, R. M. 2008, ApJ, 682, 1152

Davidsson, B. J. R. 1999, Icarus, 142, 525

de Koker, N., Steinle-Neumann, G., \& Vlček, V. 2012, PNAS, 109, 4070

Debes, J. H., Walsh, K. J., \& Stark, C. 2012, ApJ, 747, 148

Dupuis, J., Fontaine, G., Pelletier, C., \& Wesemael, F. 1992, ApJS, 82, 505

Elkin, V. G., Mathys, G., Kurtz, D. W., Hubrig, S., \& Freyhammer, L. M. 2010, MNRAS, 402, 1883

Elkins-Tanton, L. T., Weiss, B. P., \& Zuber, M. T. 2011, Earth and Planetary Science Letters, 305, 
Farihi, J. 2016, NewAR, 71, 9

Farihi, J., Jura, M., Lee, J.-E., \& Zuckerman, B. 2010, ApJ, 714, 1386

Farihi, J., Fossati, L., Wheatley, P. J., et al. 2018, MNRAS, 474, 947

Fendt, C., \& Dravins, D. 2000, Astronomische Nachrichten, 321, 193

Ferrario, L., de Martino, D., \& Gänsicke, B. T. 2015, Space Sci. Rev., 191, 111

Ferrario, L., \& Wickramasinghe, D. T. 2005, MNRAS, 356, 615

Formisano, M., Federico, C., Turrini, D., et al. 2013, European Planetary Science Congress, 8, EPSC2013

Gaillard, F., \& Marziano, G. I. 2005, Journal of Geophysical Research (Solid Earth), 110, B06204

Gänsicke, B. T., Koester, D., Farihi, J., et al. 2012, MNRAS, 424, 333

García-Berro, E., Kilic, M., \& Kepler, S. O. 2016, International Journal of Modern Physics D, 25, 1630005

Geissler, P. E., \& McMillan, M. T. 2008, Icarus, 197, 505

Ghosh, A., \& McSween, H. Y. 1998, Icarus, 134, 187

Gibert, B., Seipold, U., Tommasi, A., \& Mainprice, D. 2003, Journal of Geophysical Research (Solid Earth), 108, 2359

Giffin, A., Shneider, M., Kalra, C. S., Ames, T. L., \& Miles, R. B. 2010, ArXiv e-prints, arXiv:1004.5412

Ginzburg, S., \& Sari, R. 2016, ApJ, 819, 116

Goldreich, P., \& Lynden-Bell, D. 1969, ApJ, 156, 59

Hand, K. P., Khurana, K. K., \& Chyba, C. F. 2011, Journal of Geophysical Research (Planets), 116, E04010

Hessels, J. W. T., Ransom, S. M., Stairs, I. H., et al. 2006, Science, 311, 1901

Hevey, P. J., \& Sanders, I. S. 2006, Meteoritics and Planetary Science, 41, 95

Holsapple, K. A., \& Michel, P. 2008, Icarus, 193, 283

Johns-Krull, C. M., Greene, T. P., Doppmann, G. W., \& Covey, K. R. 2009, ApJ, 700, 1440

Jura, M. 2003, ApJ, 584, L91

-. 2006, ApJ, 653, 613 
-. 2008, AJ, 135, 1785

Kaspi, V. M., \& Beloborodov, A. M. 2017, ARA\&A, 55, 261

Kawaler, S. D. 2015, in Astronomical Society of the Pacific Conference Series, Vol. 493, 19th European Workshop on White Dwarfs, ed. P. Dufour, P. Bergeron, \& G. Fontaine, 65

Kawka, A., Vennes, S., Schmidt, G. D., Wickramasinghe, D. T., \& Koch, R. 2007, ApJ, 654, 499

Kenyon, S. J., \& Bromley, B. C. 2005, AJ, 130, 269

—. 2017a, ArXiv e-prints, arXiv:1706.08579

-. 2017b, ApJ, 850, 50

Kepler, S. O., Pelisoli, I., Koester, D., et al. 2015, MNRAS, 446, 4078

—. 2016, MNRAS, 455, 3413

Khaibrakhmanov, S. A., \& Dudorov, A. E. 2018, arXiv e-prints, arXiv:1812.10634

Koester, D. 2009, A\&A, 498, 517

Koester, D., Gänsicke, B. T., \& Farihi, J. 2014, A\&A, 566, A34

Kotera, K., Mottez, F., Voisin, G., \& Heyvaerts, J. 2016, A\&A, 592, A52

Krüger, H., Geissler, P., Horányi, M., et al. 2003, Geophys. Res. Lett., 30, 2101

Lainey, V., Arlot, J.-E., Karatekin, Ö., \& van Hoolst, T. 2009, Nature, 459, 957

Mckinnon, W. B. 1989, Nature, 340, 343

Menzel, R. L., \& Roberge, W. G. 2013, ApJ, 776, 89

Milani, A., Nobili, A. M., \& Farinella, P. 1988, Astronomische Nachrichten, 309, 38

Mohanty, S., Jankovic, M. R., Tan, J. C., \& Owen, J. E. 2018, ApJ, 861, 144

Morin, J. 2012, in EAS Publications Series, Vol. 57, EAS Publications Series, ed. C. Reylé, C. Charbonnel, \& M. Schultheis, 165-191

Mottez, F., \& Heyvaerts, J. 2011, A\&A, 532, A22

Mukherjee, D., Bult, P., van der Klis, M., \& Bhattacharya, D. 2015, MNRAS, 452, 3994

Munk, W. H. 1966, Deep Sea Research and Oceanographic Abstracts, 13, 707

Neumann, W., Breuer, D., \& Spohn, T. 2012, A\&A, 543, A141 
—. 2014, Earth and Planetary Science Letters, 395, 267

Ofir, A., \& Dreizler, S. 2013, A\&A, 555, A58

Okuzumi, S., \& Inutsuka, S.-i. 2013, in Protostars and Planets VI Posters

Opeil, C. P., Consolmagno, G. J., \& Britt, D. T. 2010, Icarus, 208, 449

Piddington, J. H., \& Drake, J. F. 1968, Nature, 217, 935

Putney, A., \& Jordan, S. 1995, ApJ, 449, 863

Rappaport, S., Sanchis-Ojeda, R., Rogers, L. A., Levine, A., \& Winn, J. N. 2013, ApJ, 773, L15

Rappaport, S., Levine, A., Chiang, E., et al. 2012, ApJ, 752, 1

Rasio, F. A., \& Ford, E. B. 1996, Science, 274, 954

Ray, J., Nagel, J., Cohrs, D., \& Rajamani, R. 2018, KONA Powder and Particle, 35, 251

Sahijpal, S., Soni, P., \& Gupta, G. 2007, Meteoritics and Planetary Science, 42, 1529

Sanchis-Ojeda, R., Rappaport, S., Winn, J. N., et al. 2014a, ApJ, 787, 47

-. 2014b, ApJ, 787, 47

Sanchis-Ojeda, R., Rappaport, S., Pallè, E., et al. 2015, ApJ, 812, 112

Scheinberg, A., Fu, R. R., Elkins-Tanton, L. T., \& Weiss, B. P. 2015, in Asteroids IV, ed. P. Michel, F. E. DeMeo, \& W. F. Bottke, 533-552

Schmidt, G. D., \& Smith, P. S. 1995, ApJ, 448, 305

Schwarz, G. 1990, Surveys in Geophysics, 11, 133161

Shimazu, H., \& Terasawa, T. 1995, J. Geophys. Res., 100, 16923

Sonett, C. P., Colburn, D. S., Schwartz, K., \& Keil, K. 1970, Ap\&SS, 7, 446

Stauffer, J., Rebull, L., David, T. J., et al. 2018, AJ, 155, 63

Taylor, G. J., Keil, K., McCoy, T., Haack, H., \& Scott, E. R. D. 1993, Meteoritics, 28, 34

Thomas, B. D., Thompson, T. G., \& Utterback, C. L. 1934, ICES Journal of Marine Science, 9, 28

Šrámek, O., Milelli, L., Ricard, Y., \& Labrosse, S. 2012, Icarus, 217, 339

van Bladel, J. 1988, IEE Proceedings A, 135, 463

Veras, D., Carter, P. J., Leinhardt, Z. M., \& Gänsicke, B. T. 2017, MNRAS, 465, 1008 
Veras, D., Leinhardt, Z. M., Bonsor, A., \& Gänsicke, B. T. 2014, MNRAS, 445, 2244

Vogel, J. K., Hascoët, R., Kaspi, V. M., et al. 2014, ApJ, 789, 75

Wickramasinghe, D. 2001, in Astronomical Society of the Pacific Conference Series, Vol. 248, Magnetic Fields Across the Hertzsprung-Russell Diagram, ed. G. Mathys, S. K. Solanki, \& D. T. Wickramasinghe, 453

Wickramasinghe, D. T., \& Ferrario, L. 2000, PASP, 112, 873

Wilson, L., \& Keil, K. 1991, Earth and Planetary Science Letters, 104, 505

Wu, Y., \& Lithwick, Y. 2013, ApJ, 763, 13

Xu, Y., Poe, B. T., Shankland, T. J., \& Rubie, D. C. 1998, Science, 280, 1415

Zuckerman, B., Melis, C., Klein, B., Koester, D., \& Jura, M. 2010, ApJ, 722, 725

Zuckerman, B., \& Reid, I. N. 1998, ApJ, 505, L143 